\title{
MISE EN ÉVIDENCE DE CRISTAUX DE CARBONATE DE CALCIUM DANS LE BOIS DES PEUPLIERS. CONSÉQUENCES SUR LA RÉPARTITION DES IONS MINÉRAUX LIÉE A LA DURAMINISATION
}

\author{
G. JANIN et A. CLÉMENT \\ Station de Recherches sur la Qualité des Bois, \\ Station de Recherches sur les Sols forestiers et la Fertilisation. \\ Centre national de Recherches forestières, I.N.R.A., \\ 54. Champenoux, par Einville
}

\begin{abstract}
RÉSUMÉ
Une recherche systématique de la présence de cristaux dans le bois du genre Populus sur de jeunes plants et des arbres adultes a été entreprise à la suite de leur mise en évidence sur les clichés radiographiques de nombreux échantillons pris sous forme de carottes de sondage.

Le phénomène de đépôts cristallisés a été étudié qualitativement et quantitativement.

L'étude qualitative a montré une présence constante de cristaux dans les sections Aigeiros et Tacamahaca et une absenoe complète dans la section Letuce. Le nombre de vaisseaux obstrués a été compté cerne par cerne à partir de coupes microscopiques sur des sections entières de Populus trichocarpa. Les modalités de répartition des dépôts dans les vaisseaux et les fibres du tronc, des branches et des racines ont été explicitées. Des analyses faites en chromatographie en phase gazeuse, en spectroscopie aux rayons $\mathrm{X}$ et en microscopie électronique classique et à balayage du type Stéréoscan ont prouvé que les cristaux étaient essentiellement formés de carbonate de calcium.

L'étude quantitative chimique sur Populus robusta et sur Populus trichocarpa des cations $\mathrm{Ca}^{++}$, $\mathrm{Mg}^{++}, \mathrm{K}^{+}$et de l'anion phosphore a permis de chiffrer cerne par cerne les concentrations atteintes et les variations observées avec la présence des cristaux.

En outre, nous avons séparé analytiquement trois groupes d'années sur chaque section :

- l'accroissement annuel voisin du cambium,

- le reste de l'aubier,

- le bois de cceur,
\end{abstract}

et nous avons mis en évidence un cerne-charnière qui sert de transition du point de vue des concentrations en ions entre le bois d'aubier et le bois de cour. 


\section{1. - INTRODUCTION}

\section{1. - Historique et observations générales sur le phénomène}

Le point de départ de la présente étude se trouve dans un rapport à distribution limitée intitulé «Première appréciation de la qualité du bois de Populus trichocarpa cultivé dans le Populetum de Velaine-Sous-Amance » (POLGe, 1967) qui contient, en outre, à titre de comparaison, une revue des qualités du bois de différents autres clones cultivés dans le même Populetum.

Le matériel végétal avait été prélevé à la tarière de Pressler sous forme de carottes de sondage et avait permis d'obtenir des images radiographiques où étaient observées les variations de la densité du bois, analysées à l'aide d'un microdensitomètre optique qui fournit un enregistrement graphique continu de ces variations (PoLGE, 1966).

Des anomalies avaient été repérées au cours de ces mêmes enregistrements : elles consistaient en des points de discontinuité sur les courbes, liés à l'existence de taches opaques aux rayons $X$ très localisées sur les clichés radiographiques. Ces variations brusques de la densité ne pouvaient pas être interprétées à l'aide des données anatomiques du végétal examiné.

Sur les radiographies, ces taches opaques aux rayons $\mathrm{X}$ se présentent comme des plages de très forte transparence où le rayonnement est complètement absorbé, ponctuelles quand I'exposition aux rayons $\mathrm{X}$ est faite parallèlement au fil du bois, sous l'aspect de bandes étroites, brillantes également, couvrant toute la largeur de la carotte quand celle-ci est radiographiée perpendiculairement au fil du bois (figure $1^{*}$ ).

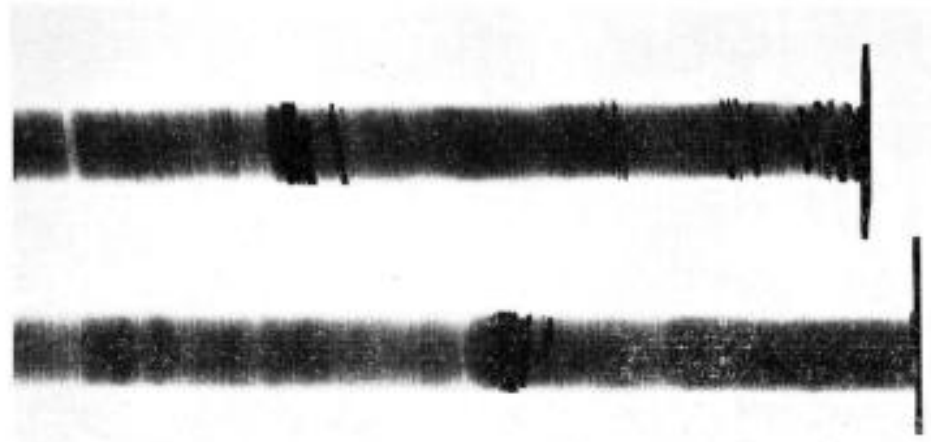

FIG. 1. - Posinif de radiographie de carottes de sondage de Populus trichocarpa montrant des traces d'inclusions minérales dans les vaisceaux $\left(\begin{array}{l}X \\ 2\end{array}\right)$

Fic. 1. - X-ray photograph positive print of Populus trichocarpa increment cores showing mineral streaks within the vessels $\left(\begin{array}{ll}X_{2} \\ 2\end{array}\right)$

On avait pu se rendre compte qu'il s'agissait d'un colmatage des vaisseaux par une substance minérale contenant probablement des sels de métaux lourds, absorbant fortement les rayons $\mathrm{X}$.

- Les cristaux apparaissent sur la radiographie elle-même sous forme de taches transparentes.

Cette figure et les suivantes de méme nature sont des images inversées oủ la présence de cristaux se traduit donc par des zones de très fort noircissement. 
Par la suite, on a pu observer le même phénomène sur tous les peupliers étudiés à la Station, quelles que soient les conditions d'environnement (Villers-Cotterêts, vallée de la Saône, vallée du Pô, Arboretum de Tervuren - Belgique), mais en revanche il ne s'est manifesté jusqu'à ce jour sur aucune radiographie des dizaines de milliers d'échantillons d'autres espèces, tant feuillues que résineuses.

Cet état de choses nous a amené à étudier plus en détail la nature de ces cristaux, leur localisation et leur importance dans la vie du végétal, notamment en rapport avec le phénomène de duraminisation dont on connait l'importance au point de vue qualité du bois.

En outre, en considérant le faible volume de bois prélevé à l'aide d'une carotte de sondage comparé au volume total de la tige, le fait que le prélèvement est effectué au hasard dans l'arbre suivant une direction radiale, et le nombre élevé de taches opaques ou de vaisseaux colmatés dans les carottes analysées, nous avons pensé qu'il s'agissait d'un phênomène suffisamment important pour mériter d'être décrit à la fois qualitativement et quantitativement.

\section{2. - Revue des travaux récents sur la présence de cristaux dans les bois feuillus et résineux}

Les ouvrages sur l'anatomie végétale et la technologie du bois ont depuis longtemps mentionné que les différents éléments du bois : vaisseaux, fibres et cellules parenchymateuses (rayons ligneux notamment) pouvaient contenir des inclusions de diverses natures, allant des variétés amorphes de substances organiques aux dépôts cristallisés de substances minérales.

Ces cristaux ont été pour la plupart signalés et identifiés dans des cas précis comme des oxalates de calcium, des carbonates de calcium ou des phosphates de calcium en mélange éventuellement avec d'autres sels (Panshiv et de Zeeuw, 1964 - Metcalfe et Chalk, 1950).

Toutefois, les dépôts cristallisés, dont fait état la littérature, sont le plus souvent décrits comme présentant un caractère accidentel et traduisant une réaction anormale du végétal.

De plus, il n'existait jusqu'ici aucune étude d'ensemble des cristaux dans le genre Populus, et nos obsarvations tendent cependant à montrer qu'ils sont présents de façon systématique dans certaines sections et que leur formation ressort d'un processus physiologique normal, comme c'est le cas pour ceux des Ulmacées (BOUrEAU, 1957).

Certains auteurs ont rattaché la présence de cristaux à des défauts ou localisations particulières du bois.

Ainsi, ReCord (1927) mentionne l'existence de cristaux de carbonate de calcium dans différentes familles et espéces de végétaux en insistant sur leur accumulation dans des sites particuliers du bois : bois de cœur, faux bois de caur, næuds, blessures, moelle et taches colorées du bois.

ELus (1967) fait porter son attention sur les relations qui peuvent exister entre la teneur en sels minéraux apportés par les dépôts cristallisés et la formation de bois de cour.

MıA (1969), dans une étude sur la coloration du bois de cour et de certaines taches ponctuelles très localisées dans le bois de l'espèce Acer saccharum, montre l'existence de nombreuses sortes de sels minéraux non identifiés contenant du calcium, du magnésium et du potassium, probablement déposés sous forme de carbonates associés à des substances phénoliques.

Nous n'avons pas eu connaissance de travaux visant à apprécier l'importance quanti- 
tative de ce phénomène pour la totalité d'un arbre étudié systématiquement, ainsi que par extension, pour l'ensemble du genre auquel cet arbre appartient.

Cependant, nous pouvons rappeler une très intéressante étude sur le genre Abies qui a été menée par des Chercheurs canadiens et américains (KENNEDY et al., 1968); dans ce travail, après une identification certaine des cristaux, en l'occurence des cristaux d'oxalate de calcium dans les rayons ligneux, ils ont procédé à des comptages en exprimant le nombre de cristaux par millimètre carré de surface de végétal sur des coupes microscopiques. Ces numérations, effectuées sur toute la hauteur d'un arbre et suivant les années, les ont conduits à suggérer une règle pratique de détermination des espèces fondée sur l'abondance et la forme des cristaux d'oxalate de calcium présents.

Au cours de tous ces travaux, les questions concernant l'origine de ces dépôts et leur rôle dans la vie de l'arbre ont été fréquemment évoquées, mais sans être complètement élucidées.

\section{3, - Buts poursuivis dans cette ètude}

Après avoir observé ces dépôts dans le bois de peuplier, nous nous sommes proposés de déterminer leur nature exacte et de décrire en détail les modalités suivant lesquelles ils se forment.

De plus, nous nous sommes efforcés de donner un aperçu des espéces du genre Populus actuellement examinées qui contiennent ou non ces inclusions pour voir si ce phénomène d'accumulation de cristaux présentait un caractère spécifique.

Enfin, nous avons cherché à exprimer quantitativement l'importance des cristallisations dans le cas de jeunes plants et d'arbres adultes au moyen de comptages et d'analyses chimiques pour préciser un aspect de l'absorption des sels minéraux par le végétal.

\section{2. - DÉTERMINATION QUALITATIVE}

Le caractère apparemment assez général de la présence de cristaux dans le bois des peupliers nous a amené à étendre nos investigations à un grand nombre d'autres espèces du genre Populus en nous intéressant aux différents aspects dendrologiques, anatomiques et physiologiques, et en procédant, enfin, à une étude physico-chimique approfondie des cristaux observés.

\section{1. - Aspects dendrologiques}

La méthode d'étude des échantillons de bois sous forme de carottes de sondage prélevées à la tarière de Pressler a facilité notre recherche. En effet, elle permet, entre autres avantages, non seulement de procéder à des tests non destructifs pour le végétal, mais encore de réunir, sur un mème cliché radiographique, de nombreux échantillons aux fins de comparaisons. C'est de ces comparaisons que ressort l'extrême variabilité du phénomène entre les différentes espèces étudiées.

\subsection{Etude sur arbres adultes}

Une revue méthodique aussi complète que possible des espèces du genre Populus dont nous disposions a été dressée en regard de la présence ou de l'absence des inclusions et de leur importance, à l'aide des peupliers cultivés dans le Populetum de Velaine-sous-Amance. 
Le tableau ci-dessous résume l'état actuel de nos observations* :

TABLEAU I

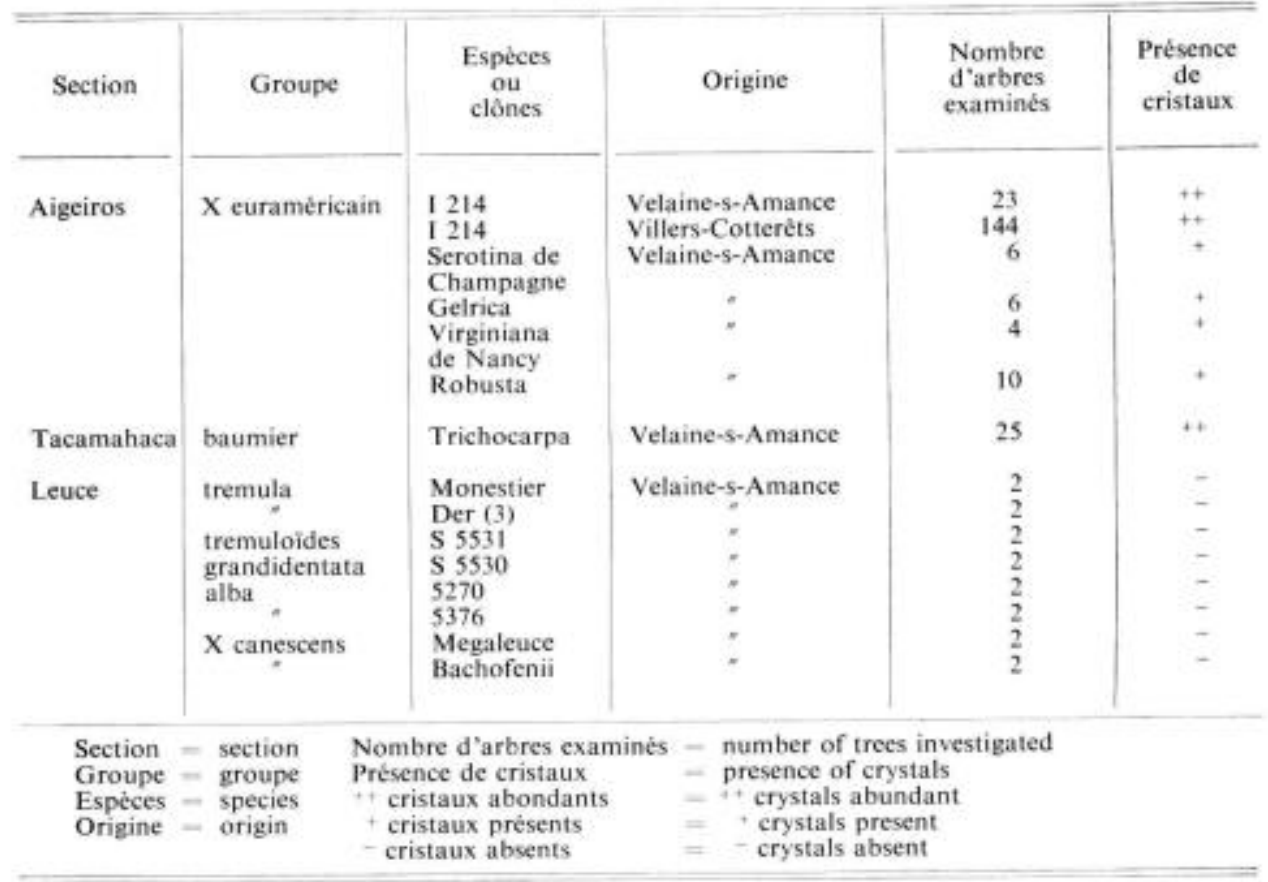

\section{Discussion}

Sur les 234 arbres examinés, nous pouvons constater :

- l'identité du comportement des espèces à l'intérieur du même groupe (toutes les espèces du même groupe dans une section possèdent ou ne possèdent pas de cristaux),

- I'opposition qui apparait entre les Aigeiros et les Leuce qui sont les sections ayant actuellement en France l'importance économique la plus grande.

De plus, il est à noter qu'un échantillonnage sous forme de carottes de sondage, malgré son faible volume relatif par rapport à celui de la tige, permet néanmoins de faire des observations valables; cela traduit l'importance et la régularité des dépôts dans les arbres.

\subsection{Etude sur jeunes plants.}

De très jeunes plants de Populus trichocarpa choisis dans le même Populetum ont été examinés; ils avaient respectivement 2 ou 3 ans et chaque entre-nœud a été radiographié.

Sur la figure 2, on peut voir, de gauche à droite, 3 jeunes plants : l'un de 3 ans et les autres de 2 ans, et, placées les unes au-dessus des autres, les sections de $5 \mathrm{~mm}$ d'épaisseur correspondant à chacune des pousses en hauteur. Sur la section inférieure du plant de 3 ans, on aperçoit des points noirs très contrastés localisant les cristaux de carbonate de

(*) Ce tableau sera complété dans un travail ultérieur; nous pouvons déjà citer que, sur des peupliers en provenance d'Alep (Syrie), des cristaux ont été trouvés dans Populus nigra (L.) c. v. Hamoui, et que leur absence a èté confirmée sur Populus alha (L.) c. v. Roumi. 


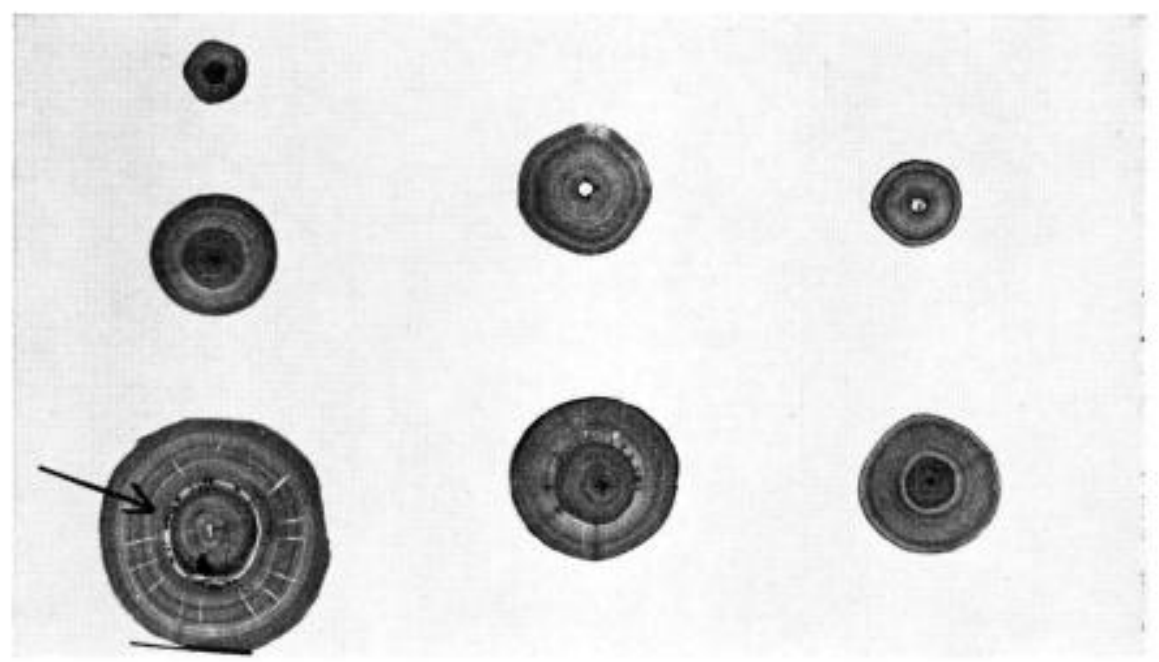

FiG. 2. - Positif de radiographie des entre-naruds de 3 jeanes plants de Populus trichocarpa de 3 ans et de 2 ans. La fleche indique la présence de cristawx dans le premier entre-newd (grandeur nature)

FiG. 2, - X-ray radiogroph positive print of internodes of three youngs plants of 3 and 2 years old plants of Populus trichocarpa. The arrow shows the crystals in the first internode (original size)

Le tableau ci-dessous résume les observations qui ont pu être faites :

\begin{tabular}{|c|c|c|c|}
\hline $\mathrm{N}^{\infty}$ plants & Iur entre-naxud & $2+$ entre-naud & 3 entre-noud \\
\hline $\begin{array}{l}1 \\
2 \\
3\end{array}$ & $\begin{array}{l}\text { néant } \\
\text { néant } \\
\text { néant }\end{array}$ & $\begin{array}{l}\text { néant } \\
\text { néant } \\
\text { néant }\end{array}$ & cristaux abondants \\
\hline
\end{tabular}

calcium. En revanche, ceux beaucoup plus diffus qui apparaissent sur la section inférieure du $2^{\mathrm{e}}$ plant ne sont pas de même nature et pourraient correspondre à des accumulations de composés chimiques amorphes non encore identifiés.

Chronologiquement, la formation des dépôts cristallisés commence donc très tôt dans la vie de la plante, puisqu'on en observe dès la troisième année de pousse dans ce que l'on peut appeler le cocur du jeune arbre.

La figure 3 concerne 2 autres niveaux de l'entre-nceud inférieur de la tige $\mathrm{n}^{*} 1$; on y retrouve les mêmes accumulations de cristaux, ce qui prouve la régularité des dépôts.
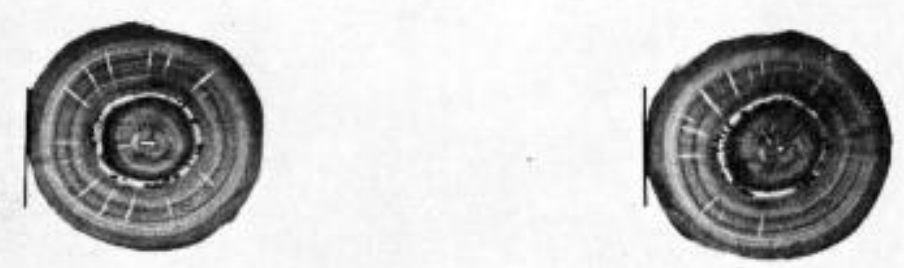

FıG. 3. - Positif de radiographie montrant des cristaux à deux niveaux différents dans le mëme entre-naud (grandear nature)

FıG.-3, - X-ray radiograph positive print showing crystals at two different levels in the same internode (original size) 
Ces observations seront corroborées par des analyses minérales quantitatives dans la suite de cet exposé.

\section{2. - Aspects anatomiques}

Les dépôts de cristaux ont été observés jusqu'ici dans les vaisseaux et dans les fibres de la tige principale et aussi dans les branches et les racines,

\subsection{Localisation à Tintérieur des accroissements.}

\subsection{Dans la tige principale.}

L'examen de clichés radiographiques d'échantillons pris dans la planche de cœur à différents niveaux dans l'arbre montre que des dépôts existent dans tout l'arbre (figures 4 et 5 ).

La localisation de ces cristaux se fait suivant deux modalités :

- présence constante au cour à tous les niveaux à l'intérieur d'un cylindre central limité aux deux premiers cernes de chaque entre-nœud, excepté dans les dernières pousses annuelles,

- présence aléatoire, fonction de la distance à partir du coeur et du niveau de prélèvement, à l'intérieur d'un cône n'intéressant pas les derniers accroissements produits.

Pour mieux décrire ce mode de dépôts quantitativement, nous avons dénombré tous les vaisseaux obstrués sur plusieurs sections transversales entières d'un Populus trichocarpa de $25 \mathrm{~m}$ de haut, respectivement à $3 \mathrm{~m}, 9 \mathrm{~m}$ et $18 \mathrm{~m}$. Nous avons divisé la surface de chaque rondelle en rectangles de $6 \times 1 \mathrm{~cm}$ sur lesquels nous avons prélevé une coupe mince de 20 à $25 \mu$ d'épaisseur.

En utilisant avec une lumière rasante et modérée une loupe binoculaire de grossissement $\times 40$, nous avons pu, grâce à la différence de réfringence entre la cellulose et les cristaux, localiser et compter cerne par cerne les vaisseaux obstrués. Nous avons, par ailleurs, calculé la surface de chacun des accroissements correspondants pour obtenir une densité moyenne par unité de surface pour chaque année (voir annexe 1 et figures 6 et 7).

\section{Discussion}

On peut observer que les accroissements annuels au voisinage du cambium, là où la circulation de la sève est la plus importante, sont dépourvus de cristaux.

De plus, si la densité des dépôts diminue régulièrement du cauur vers l'écorce du fait de l'augmentation de la surface des cernes successifs (voir figure 6), le nombre des vaisseaux obstrués peut augmenter ou diminuer au hasard d'année en année et d'un niveau à l'autre (voir figure 7).

Ces deux remarques nous montrent que le nombre de cristaux déposés n'est pas lié à la rapidité de croissance de l'année considérée, mais qu'il s'agit d'un phénomène-retard progressif de dépôts de beaucoup postérieur à l'année de végétation. 


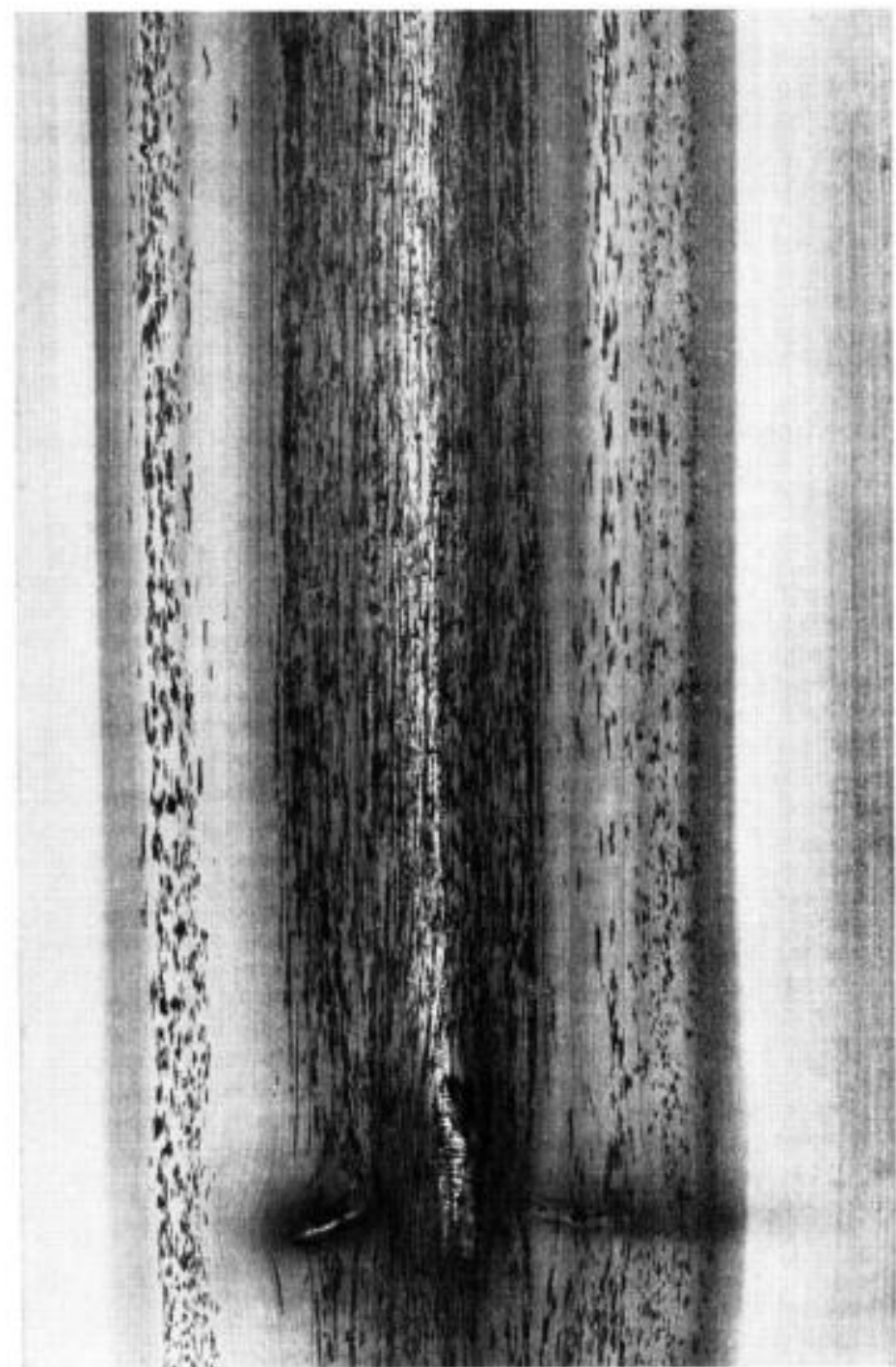

Fig. 4. - Poshif de radiographie d'une planche de caur de $10 \mathrm{~mm}$ d'épaissear de Populus trichocarpa prise à une hauteur de $10 \mathrm{~m}$. Lex incluxions minérales sont encore trés importantes $(X 2)$

F1G. 4. - X-ral photogzoph positive print of hearlwewd board ten millimeters in thickness taken at the height of to meters. The mineral streaks are yet very important ( $\left.\begin{array}{l}X \\ 2\end{array}\right)$

\subsection{Dans les branches et les racines.}

Pour avoir une vue d'ensemble de toutes les parties de l'arbre, nous avons radiographié des sections de branches, prises à une fourche par exemple, et des sections de racines de différents diamètres, et sur chaque radiographie nous avons pu constater la présence de cristaux dans les vaisseaux. 


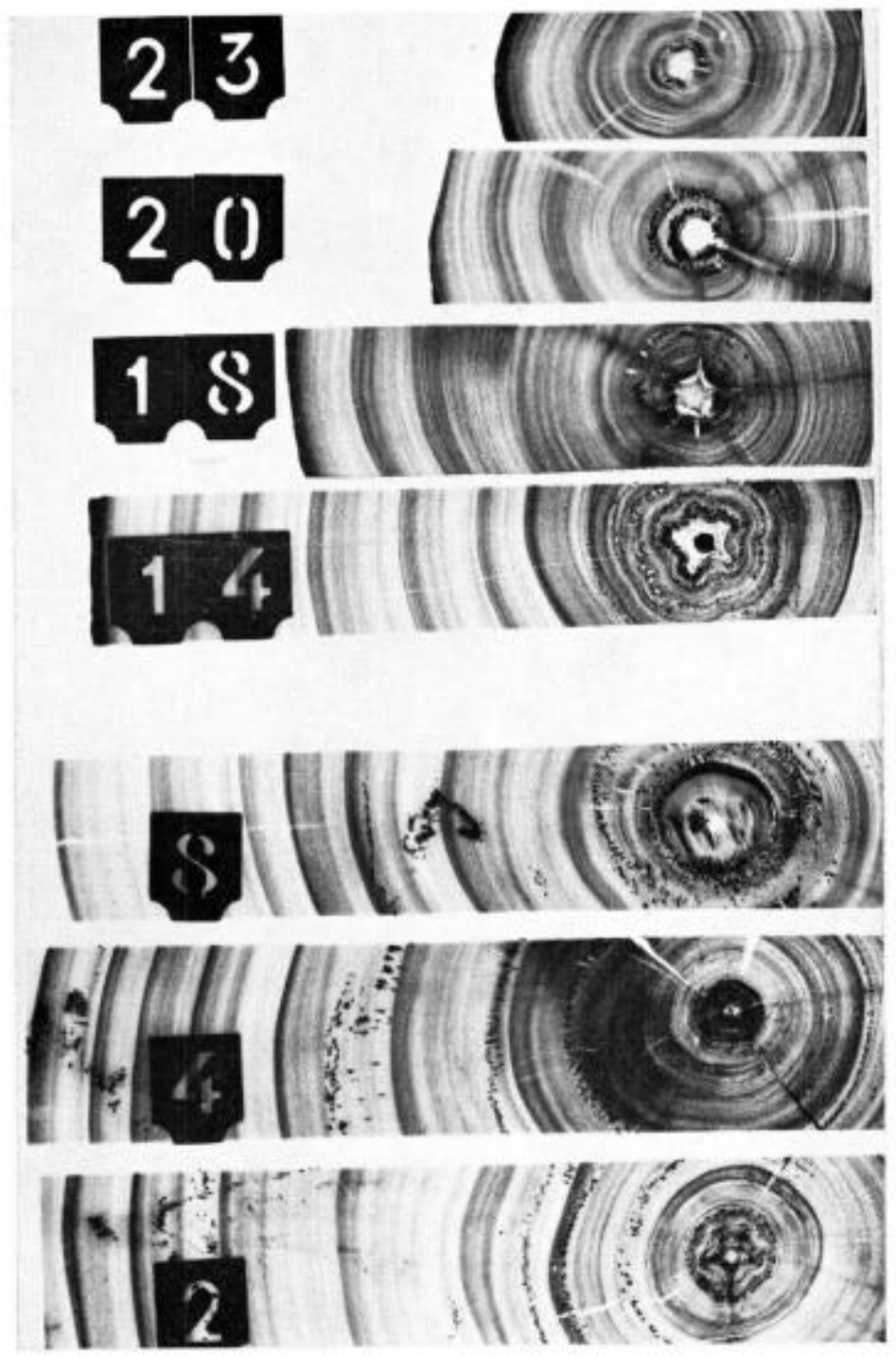

Fig. 5. - Pasinif de radiographie de planches radiales de $10 \mathrm{~mm}$ d'dpaisseur de Populus trichocarpa prises à $2,4,8,14,18,20$ ef 23 mères de hauteur. Noter Tahondance et la locafisarion particulière des dépôts dans les planches $2,4,8$ (grandesur nature)

FIG. 5. - Radiograph positive print of radials boards ten millineter in thichness of Populus trichocarpa taken at $2,4,8,14,18,20,23$ meters height. Note the abundance and the particular localisation of the deposits in the boords 2, 4,8 (original size)

\subsection{Localisation à Tintérieur des cellules.}

L'examen de toutes les coupes transversales radiographiées montre que les zones de bois initial et de bois final sont indifféremment affectées. Parfois, une bande continue de vaisseaux obstrués peut même chevaucher une limite de cernes. 


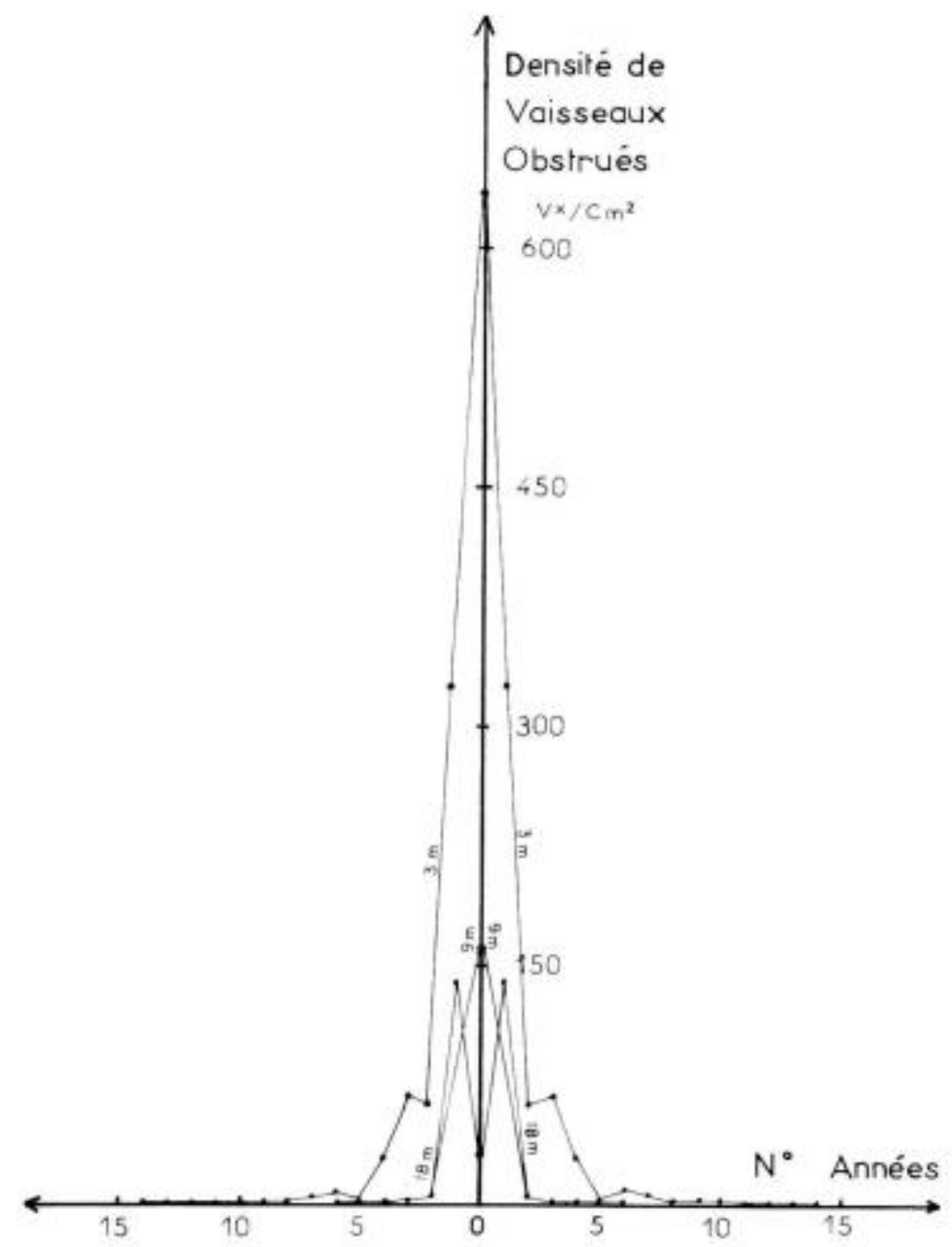

Fig. 6. - Densité des vaisseaux obstrués dans Populus trichocarpa aux nìveaux 3, 9, 18 mètres pour chaque année du caur vers l'ecorce

Fic. 6. - Density of the filled vessels in Populus trichocarpa at levels 3,9, 18 meters for each year from the heartwood to the hark

En se reportant à la figure 5 , on peut noter sur l'échantillon en coupe transversale prélevé à 2 mètres (2) des dépôts circulaires, aussi bien dans le bois initial que dans le bois final. Pour l'échantillon pris au niveau de 8 mètres (8), les vaisseaux obstrués recouvrent la limite de deux années consécutives. Ceci confirme que les précipitations interviennent bien après la formation des accroissements annuels en cause.

\subsection{Mode de dépóts dans les vaisseaux.}

Sur des coupes transversales effectuées au microtome et observées à la loupe binoculaire $(\times 40)$, les vaisseaux présentent, soit un tapissage interne, soit un remplissage partiel, 


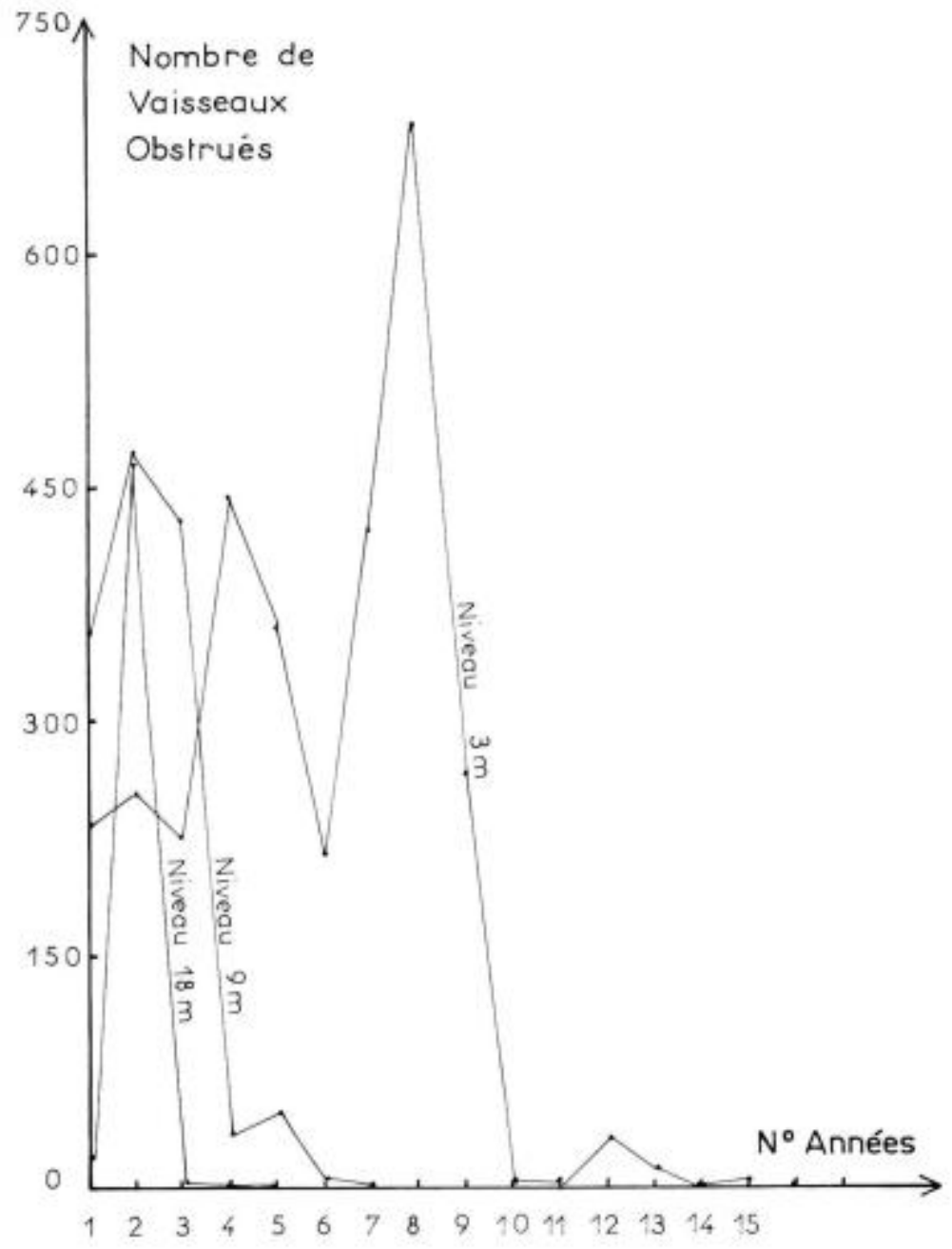

F1G. 7. - Variation du nombre de vaisseaux obstrués dans le tronc de Populus trichocarpa aux niveaux 3,9, 18 metres et pour chaque année du cour vers l'écorce

FIG. 7. - Variation in number of the filled vessels in the bole of Populus trichocarpa at the levels 3 , 9. I8 meters and for each year from heartwood to the bark

comme lors d'un début de dépôt, ou bien dans le cas le plus fréquent, un colmatage complet; à plus fort grossissement, en effet, on peut observer un véritable moulage du vaisseau en vraie grandeur (figure 8), et les empreintes de deux ponctuations du vaisseau qui apparaissent sur un fragment de cristal (figure 9) montrent bien que la cavité se trouvait intégralement remplie.

La remarque la plus importante qui puisse être faite est que les vaisseaux obstrués ne sont jamais endommagés et ne correspondent pas à des zones de bois attaquées ou en voie 


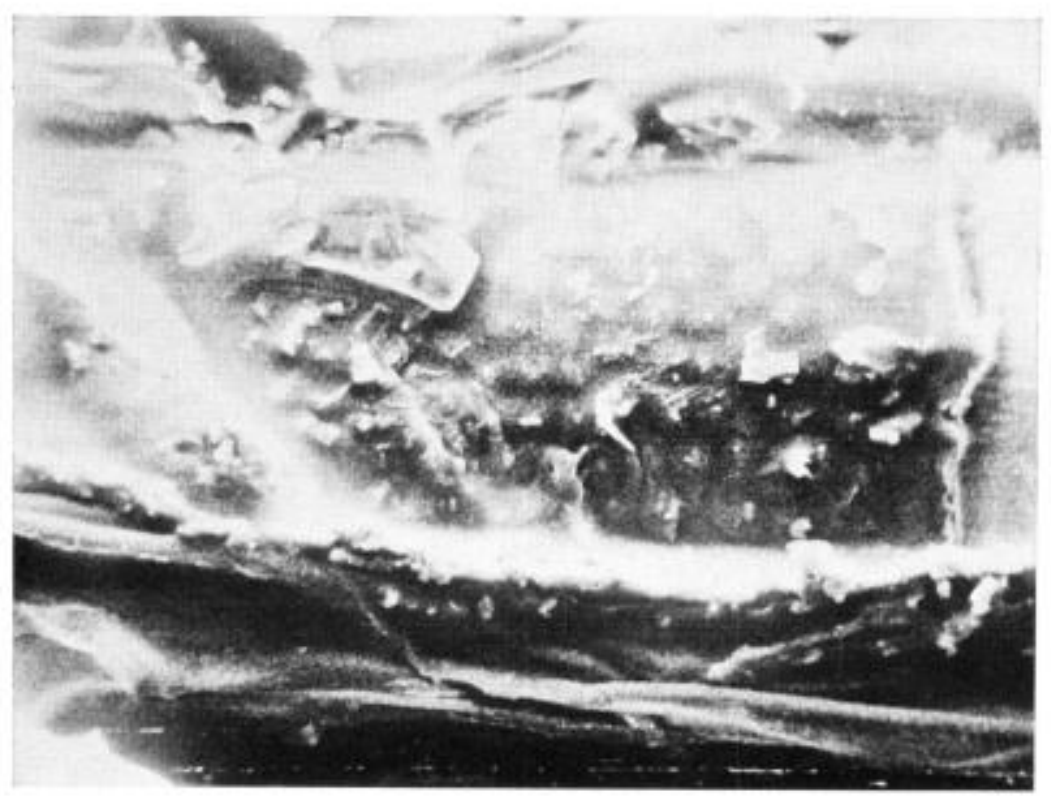

F1G. 8. - Sur la photo prise au microscope electronigue Stéréoscan, on peut voir une partie de vaisseau completement reanpli avec ses ponctuations en relief $(X 3000)$

FiG, 8. - Scanning electron microscope photograph showing a part of a full-filled wessel with its pits rellef $(X 3000)$

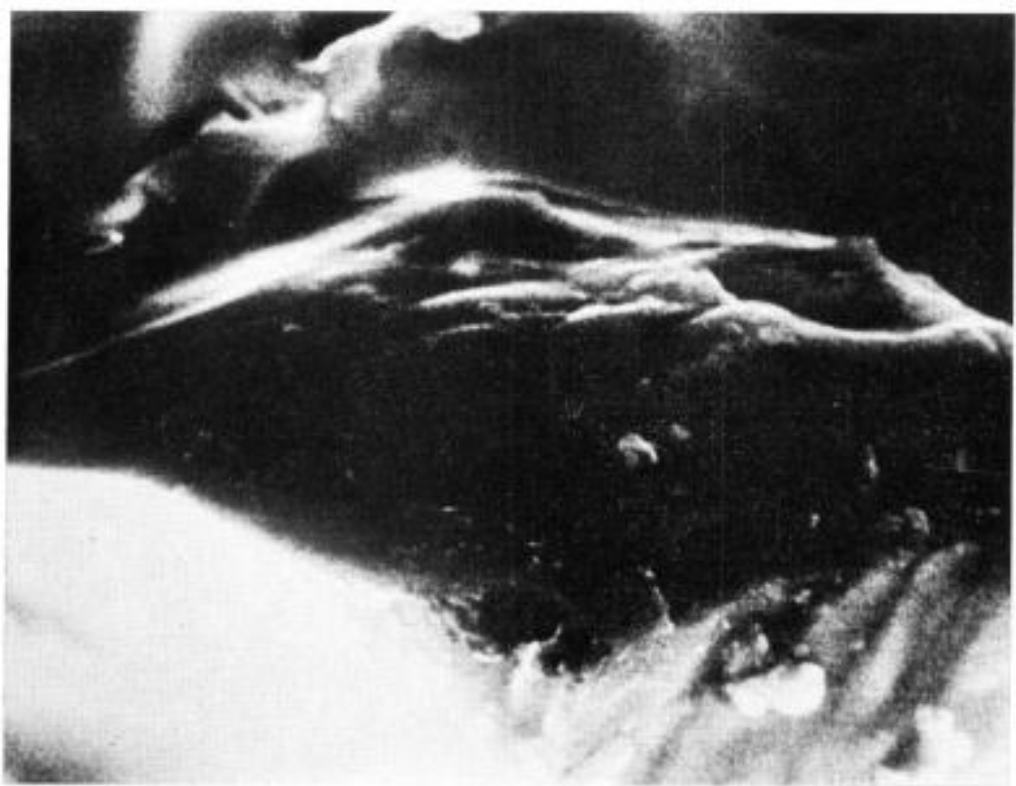

FiG. 9. - Surface détaillée d'sun fragment de cristal montrant lempreinte de deax ponctuations $(X 12000)$ Fio. 9. - Detailed surface of a crystal chip showing the print of two pits ( $X 12000)$ 
de destruction. Elle corrobore l'idée que la présence de ces cristaux est un des aspects de la physiologie normale de l'arbre (voir figures 10 et 11).

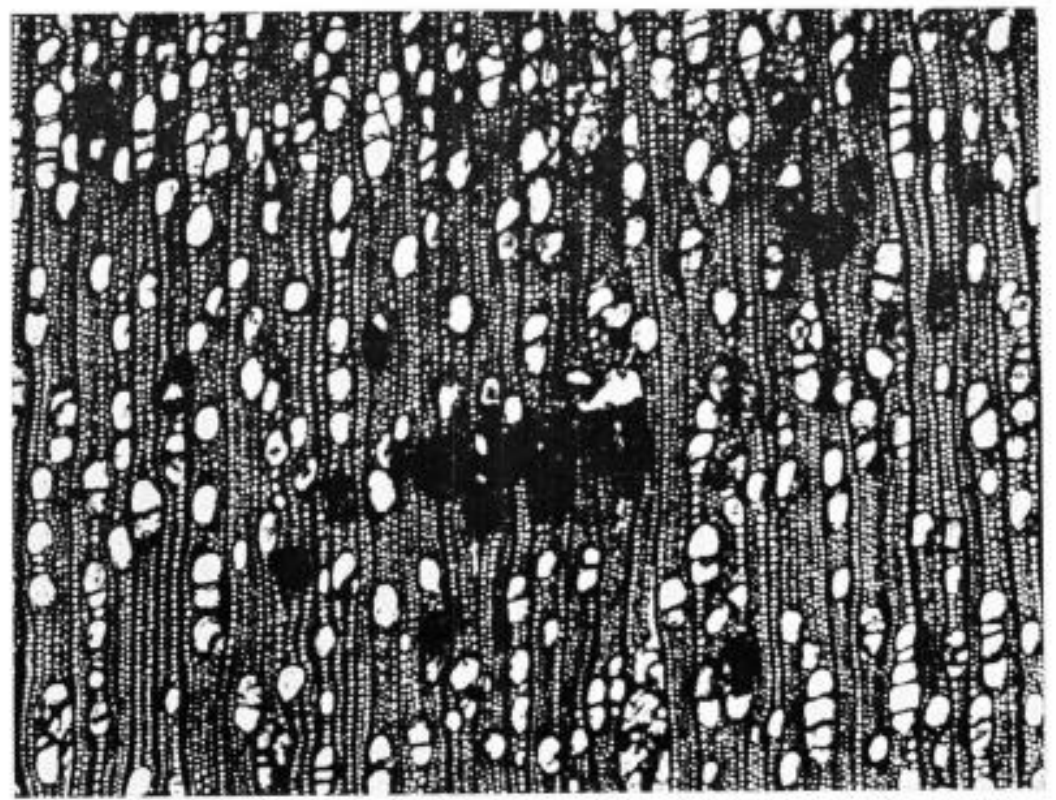

Fio, 10. - Coupe transwersale montrant la répartition des vaisseaux obstrués dans le bois d'aubier normal de Populus trichocarpa $(X+0)$

Fic. 10. - Transverse section showing the distribution of filled vessel in the normal sapwood of Populus trichocarpa $(X+0)$

Le remplissage de ces vaisseaux freine la circulation de la sève dans la région des dépôts. En effet, comme on peut le voir sur la figure 4, les vaisseaux remplis se trouvent décalés dans le sens longitudinal les uns par rapport aux autres et bloquent, de proche en proche, le passage de la sève. Cet écoulement lent de la sève peut favoriser, par un phénomène de saturation, les précipitations et contribuer à augmenter les dépôts cristallisés.

Nous avons pa constater, dans chaque cas, après dissolution des cristaux dans l'acide chlorhydrique $\mathrm{N} / 10$, que toutes les membranes des vaisseaux étaient intactes. De plus, au cours de cette attaque, nous avons pu apercevoir un dégagement gazeux qui sera étudié plus loin.

\subsection{Mode de dépôts dans les fibres.}

Un examen attentif des coupes a permis de voir, autour des vaisscaux colmatés, des groupes de fibres colmatées elles aussi. Dans ce cas, on peut penser que les ponctuations des vaisseaux en anastomose avec les ponctuations des fibres laissent passer, de proche en proche, la sève et les substances qui précipitent et contribuent ainsi à étendre la zone de colmatage (voir figure 12). 


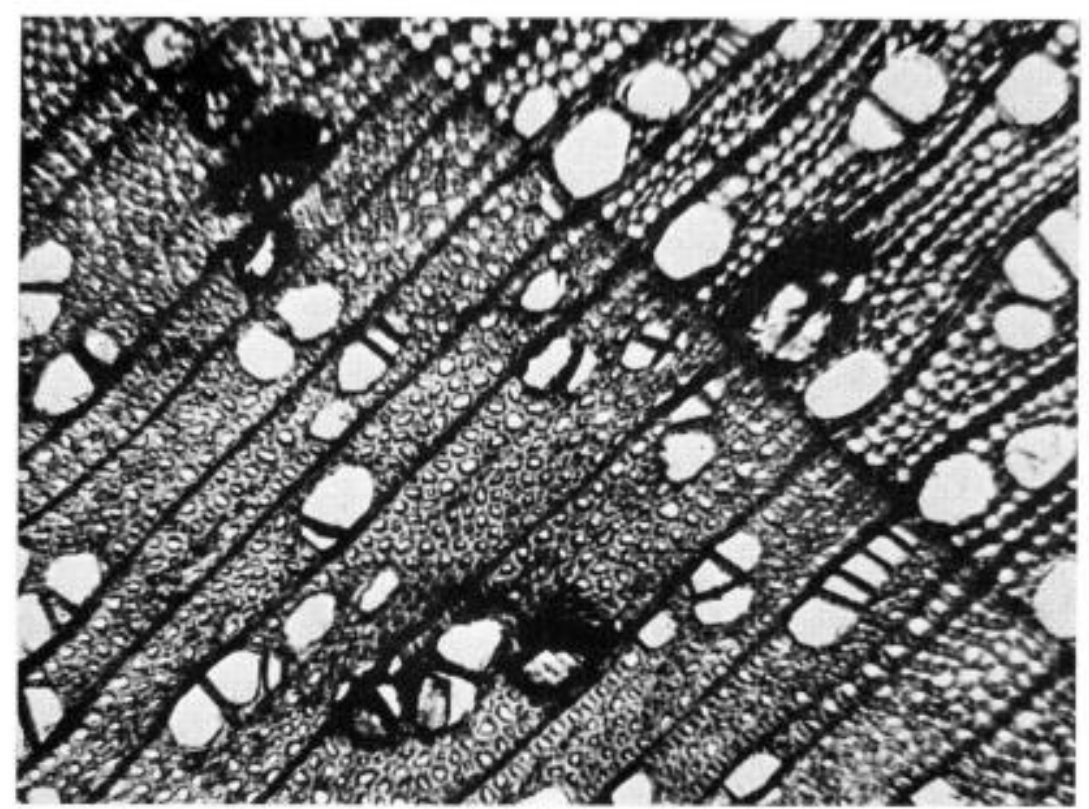

Fio. 11. - Coupe transwersale montrant sue la gauche des cristaux dans des vaisseaux du bois final, et en haut à droite dans un waisseau du bois initial près de la limite de cerne ( $\left.X_{120}\right)$

FiG. 11. - Transverse section showing on the left crystals within vessels of latewood and on the right within vessel of earlywood clase to the limit of two rings $(X \mid 20)$

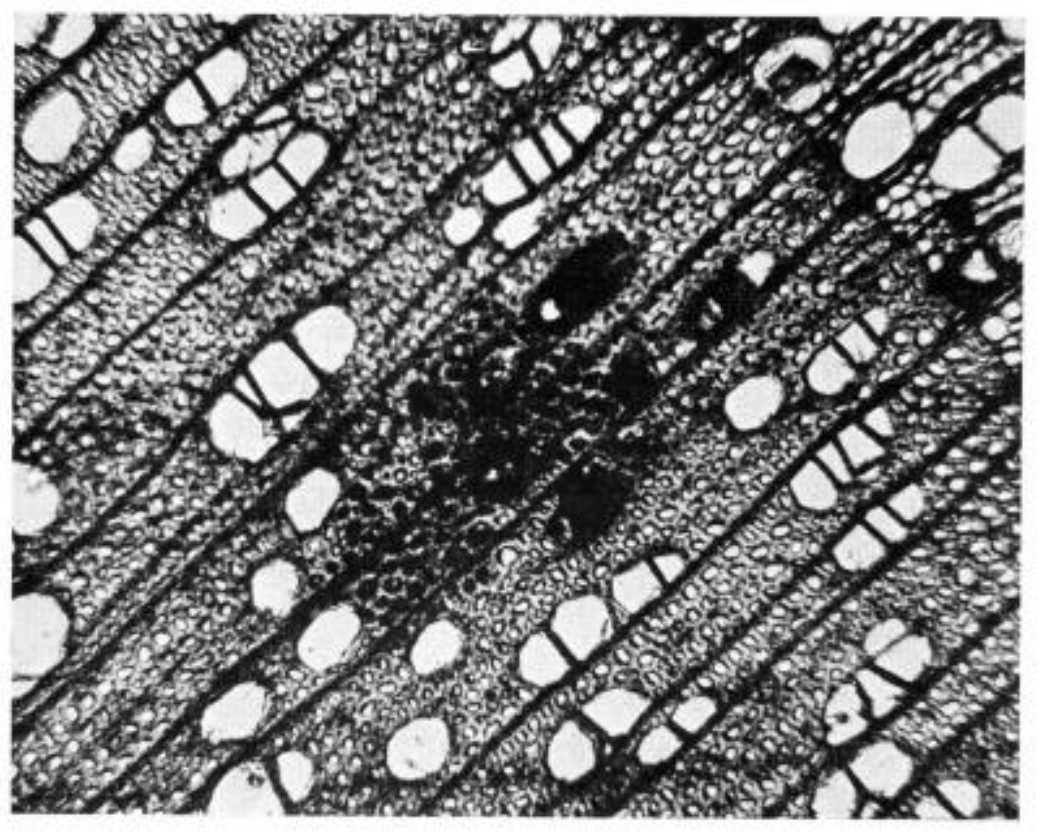

FIG. 12. - Coupe tranxversale montrant des vaisseaux ef des fibres remplis avec les mémes cristaux $(X 120)$

Fui. 12. - Transverse section showing vessels atd fibers filled with the same crystals $(X 120)$ 
L'essai de dissolution des cristaux par l'acide chlorhydrique a êté repris. II a laissé apparaitre les fibres intactes. Au cours de cet essai, nous avons observé l'apparition lente mais continue de gaz localisé au-dessus de chaque fibre, comme dans le cas des vaisseaux, ce qui tend à montrer que les vaisseaux et les fibres sont colmatés par la même substance.

\section{3. - Méthodes physiques et physico-chimiques mises en auve pour déterminer la nature chimique des cristaux}

La quantité exceptionnellement faible de précipité contenu dans chaque vaisseau obstrué et l'extrême difficulté de sa récupération ont rendu les déterminations très délicates et nous ont conduit à employer plusieurs méthodes de microanalyse, parfois à la limite de leurs possibilités d'utilisation.

\subsection{Chromatographie en phase gazeuse.}

\subsection{Choix de la méthode.}

Nous avons été amenés à choisir la chromatographie à la suite d'une observation faite sur des coupes minces de bois contenant des inclusions.

Dans un premier temps, nous avions recherché les oxalates parce que le calcium avait été signalé sous cette forme dans le végétal par de nombreux auteurs. Nous avons utilisé la méthode de R. W. KENNEDY et al. (1968, op. cit.); cette recherche s'est révélée négative.

C'est au cours de cette manipulation que nous avons observé sous le microscope binoculaire un dégagement gazeux sur les vaisseaux et les fibres contenant les substances opaques.

C'est ce dégagement gazeux, sous forme de bulles très fines fixées à la coupe du végétal, qui nous a donné l'idée de tenter une analyse par chromatographie en phase gazeuse, à la condition d'obtenir un dégagement en volume suffisant pour effectuer les prélèvements nécessaires à la manipulation.

A ce moment, nous avons pensé que les dépồts pouvaient être formés de carbonate de calcium qui, en milieu aqueux acide, donnerait par réaction ionique :

$$
\mathrm{CaCO}_{3}+2 \mathrm{HCl} \rightarrow \mathrm{Ca}^{++}+2 \mathrm{Cl}^{-}+\mathrm{H}_{2} \mathrm{O}+\mathrm{CO}_{2}^{+}
$$

\subsection{Matériel et mode opératoire.}

Nous avons utilisé des coupes longitudinales de Populus trichocarpa de 20 à $25 \mu$ d'épaisseur prélevées dans les parties du cceur de l'arbre contenant le plus d'inclusions. Un gramme de végétal fournit en moyenne 250 à 300 de ces coupes; pour chaque détermination, nous avons utilisé $3 \mathrm{~g}$ environ de bois, ce qui, compte tenu de la solubilité du $\mathrm{CO}_{2}$ dans l'eau, nous a permis d'obtenir un dégagement gazeux suffisant de 30 à 35 microlitres.

Dans un bécher d'eau distillće de 2 litres, après ébullition prolongée, nous avons soumis l'eau et les coupes minces à un dégazage vigoureux dans un dessicateur de grandes dimensions pour éliminer I'air dissout dans le liquide et occlus dans les vaisseaux du végétal.

Au bout de 30 minutes, le dégazage terminé, nous avons recouvert les coupes immergées et saturées avec un entonnoir muni d'une tige très fine et dont l'extrémité est obturée, après immersion, par une pastille découpée dans du caoutchouc de silicone du type septom, utilisée sur les appareils de chromatographie en phase gazeuse pour assurer l'étanchéité des orifices d'injection des échantillons.

Après addition d' $\mathrm{HCl} 2 \mathrm{~N}$ au contact des coupes, les bulles formées se dégagent lentement et viennent se rassembler sous le bouchon de caoutchouc où elles sont retenues. Elles peuvent être alors prélevées à l'aide d'une seringue Hamilton de 10 microlitres. 
Nous avons pratiqué ces analyses de chromatographie en phase gazeuse à l'École Nationale Supérieure des Industries Chimiques de Nancy, au département de Génie Chimique, sous la direction de M. Sutre, Ingénieur du C.E.R.C.H.A.R.

Le chromatographe utilisé était un Perkin Elmer F 73 T dont le système de détection est assuré par une thermistance. Les caractéristiques de fonctionnement étaient les suivantes :

- gaz vecteur : Hydrogène au débit de $72 \mathrm{~cm}^{3}$ par minute.

- température du four : $100^{\circ}$,

- sensibilité de la détection pour toute l'échelle du chromatographe : $10 \mathrm{mV}$ pour les injections d'air et de $\mathrm{CO}_{2}$ technique, et $2,5 \mathrm{mV}$ pour le gaz des échantillons de peupliers,

- volume de gaz injecté à chaque mesure : 10 microlitres,

- vitesse d'enregistrement du graphospot : 30 millimètres par minute.

La colonne de un métre de longueur était remplie par du gel de silice pour séparer l'air du CO:

Compte tenu de notre hypothèse de départ, nous avons choisi pour étalonner la colonne et déterminer les temps de rétention des gaz :

- le gaz $\mathrm{CO}_{2}$ technique qui contient $99,3 \%$ de $\mathrm{CO}_{2}$ et $0,7 \%$ d'air,

- l'air ambiant.

Les temps de rétention étaient respectivement, dans l'ordre des passages :

- pour l'air : 20 secondes, soit 10 millimètres sur le graphique,

- pour le $\mathrm{CO}_{2}$ : 106 secondes, soit 53 millimètres sur le graphique.

Ils sont repérés du point d'injection des gaz au maximum du signal correspondant (figure 13).

\subsection{Résultats.}

Sur la figure ci-après sont placés, côte à côte, les chromatogrammes correspondant :

1) aux injections d'air ambiant,

2) aux injections de gaz carbonique technique,

3) aux injections de " gaz » de peuplier.

La comparaison de l'ordre de sortie des composés du chromatogramme n ${ }^{\circ} 3$ et l'identité des temps de rétention de ces composés repérés sur les chromatogrammes $\mathrm{n}^{03} 1$ et 2 permettent d'affirmer qu'il s'agit bien d'air et de gaz carbonique.

Le prélèvement gazeux recueilli au-dessus des coupes de végétal contient du $\mathrm{CO}_{2}$ principalement. Il provient bien de la dissolution des cristaux. L'importance du « pic » montre que la majeure partie des sels déposés se trouvent sous forme de carbonates.

Malgré plusieurs essais, il n'a pas été possible d’éliminer totalement l'air résiduel qui provient de la méthode de prélèvement et d'injection des échantillons.

Les résultats obtenus par la chromatographie dans ce cas précis ne peuvent être que qualitatifs, car l'impossibilité de peser directement les cristaux ne permet pas de ramener le volume de gaz dégagé au poids de cristaux utilisés pour le dégagement.

Cette méthode nous a permis de vérifier qu'il s'agissait bien de carbonates, mais il nous a fallu utiliser d'autres méthodes pour recouper ces résultats et reconnaitre la présence éventuelle d'autres sortes de cristaux.

Tel est le but des études aux rayons $\mathrm{X}$ et en microscopie électronique que nous avons réalisées à l'École des Mines, dans le Laboratoire de Métallurgie de M. le Professeur FAIVre, sous la direction de M. Gantors. 


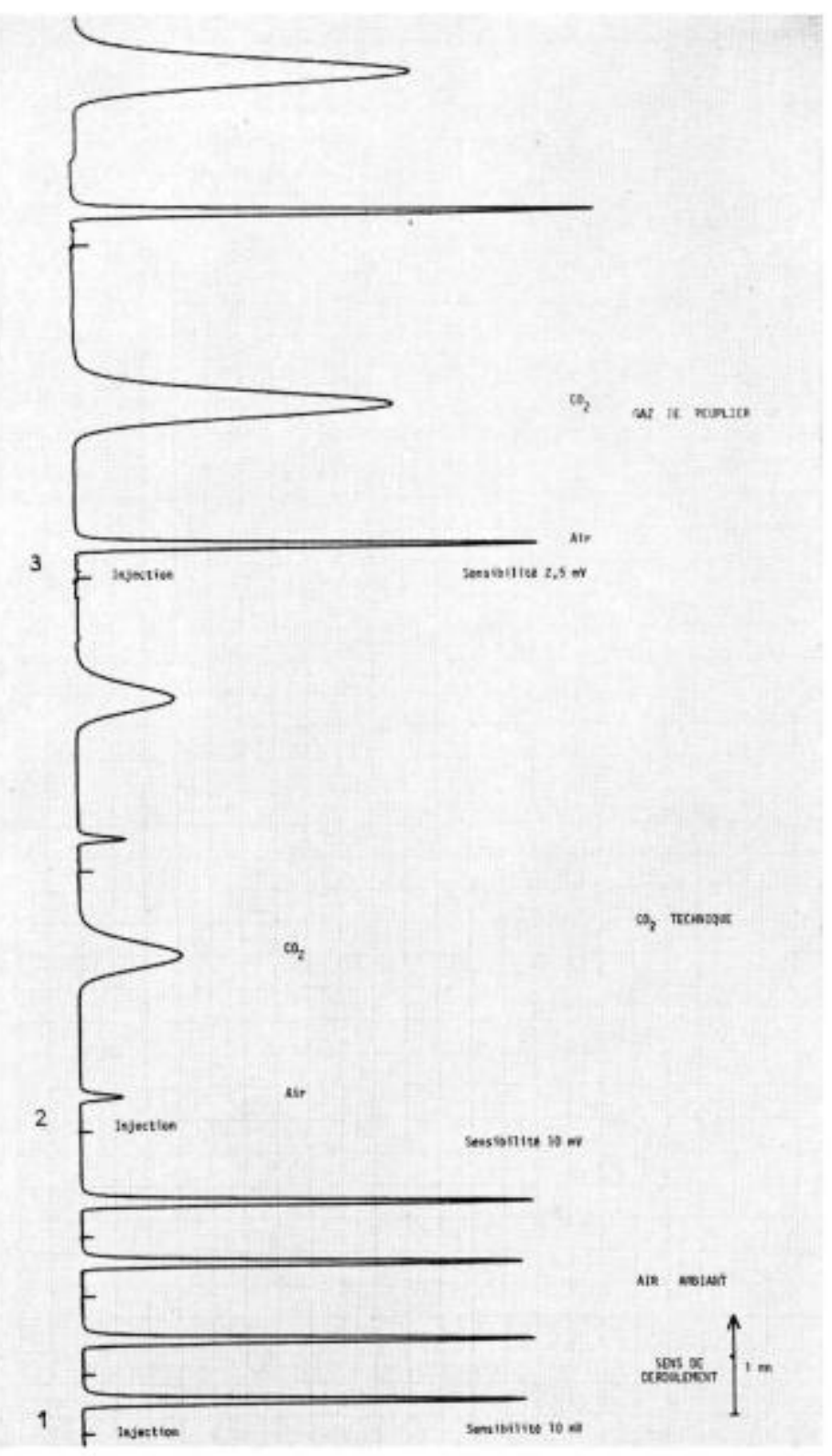

FıG. 13. - Diagramme de chromatographie montrant Fidentification du gaz dégagé à partir des cristaux situés dans les coupes de bois de peuplier (3); Vair (1) et le $\mathrm{CO}_{2}$ technique $(2)$ ont été choisis comme gaz de référence.

FiG. 13. - Chromatographic record showing the determination of the nature of gas relaesed from crystals included in sections of Populus (3); the air $(I)$ and the technical $\mathrm{CO}_{2}(2)$ are selected as gas reference. 


\subsection{Diffraction des rayons $X$.}

\subsection{Préparation des échantillons et mode opératoire.}

L'extrềme petitesse des dépôts sur une coupe microscopique ne permet pas de soumettre aux rayons $\mathrm{X}$ une seule coupe car l'intensité des rayons diffractés serait alors trop faible.

Nous avons donc écrasé sur une surface adhésive - un ruban autocollant - un grand nombre de vaisseaux colmatés prélevés sur une série de coupes minces pour constituer une plage recouverte de cristaux en quantité suffisante, soit une plaque de $2 \mathrm{~cm}$ de long sur $1 \mathrm{~cm}$ de large.

Les résultats de l'analyse chromatographique nous avaient guidé dans le choix des substances témoins à soumettre en méme temps que notre échantillon aux rayons $\mathrm{X}$ comme substances étalons. Nous avons retenu le carbonate de calcium, le carbonate de magnésium et le carbonate de potassium.

Les clichés de la figure 14 ont été obtenus par transmission selon le montage Guinier-SeemannBohlin avec une chambre multiple Eraf Nonius et un générateur C.G.R. $\theta$ 50, d'une puissance de $500 \mathrm{~W}$, soit en fonctionnement, pour un courant de $10 \mathrm{~mA}$, une tension de $50 \mathrm{kV}$. Les échantillons ont été soumis à 15 heures d'exposition.

\subsection{Résultats.}

Sur la figure 14 :

- le cliché $n^{\circ} 1$ rassemble dans l'ordre, de gauche à droite, les spectres des carbonates de potassium (a), de magnésium (b), de calcium (c).

- Ie cliché $n^{\circ} 2$ montre, de gauche à droite, les spectres du carbonate de calcium cristallise (d) et des cristaux extraits du bois de peuplier (e) et (f).

La comparaison entre les spectres (c) et (d) d'une part, et les spectres (e) et (f) des cristaux étudiés, montre que ceux-ci sont formés en majeure partie de carbonate de calcium.

Cependant, il existe des raies supplémentaires qui, comme dans beaucoup de cas, peuvent être dues, soit au support organique : film plastique et colle, soit à la présence d'autres substances cristallisées en quantité plus faible et qui, malgré des temps d'exposition très longs, donnent des raies de faible intensité.

\subsection{Microscopie électronique.}

A l'aide d'un microscope électronique Philips E. M. 300, nous avons obtenu des clichés de diffraction électronique sur les mêmes cristaux déposés sur des grilles fines recouvertes d'un film de collodion. Ce microscope possède une platine goniométrique qui permet de prendre des clichés du même cristal sous différentes orientations.

Par opposition avec les spectres de diffraction aux rayons $\mathrm{X}$ qui intègrent la diffraction d'un grand nombre de cristaux et donnent un effet global qui rend compte de la présence du constituant le plus important, la microscopie électronique permet d'atteindre chaque cristal susceptible de diffracter. Aussi, avons-nous obtenu, au cours de cette étude, de nombreux clichés de diffraction de substances inconnues.

La détermination de ces substances sera intéressante à faire pour connaitre les associations de tous les minéraux existant dans ces cristaux. Elle fera l'objet d'études ultérieures plus complètes. Nous pouvons signaler dès maintenant que leur importance relative est faible par rapport à celle du carbonate de calcium. 


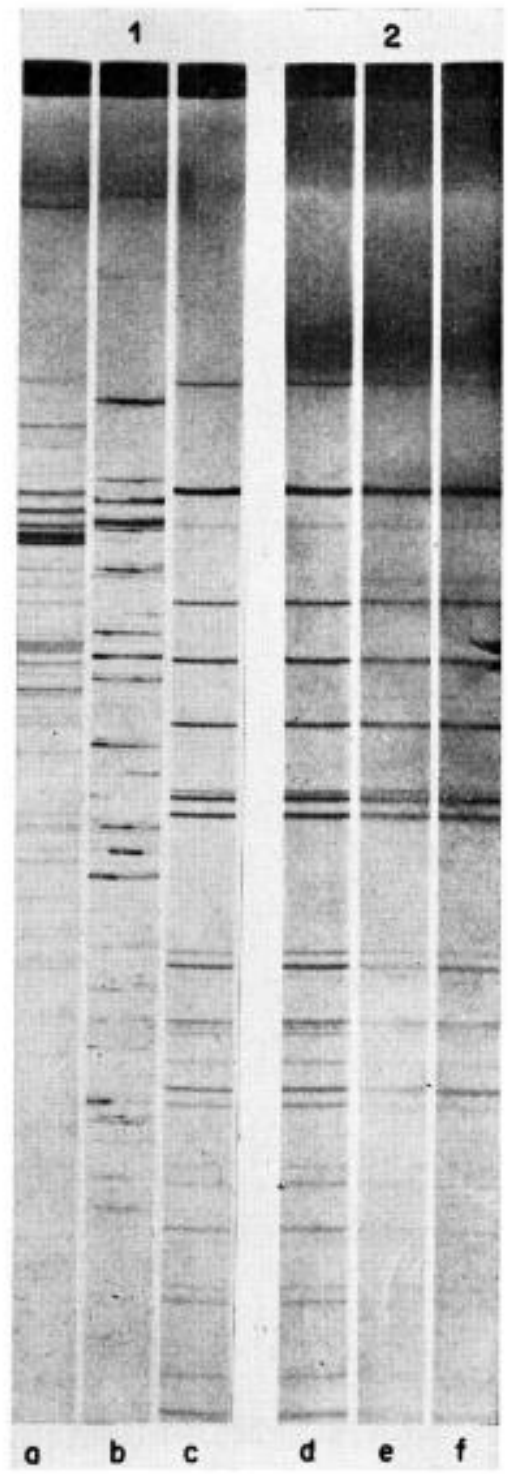

Fig. 14. - Positif de spectres de diffraction

1) produits purs cristallisés de référence : a $\mathrm{K}_{2} \mathrm{CO}_{2}$

b $\mathrm{M}_{g} \mathrm{CO}_{\text {, }}$

c $\mathrm{CaCO}_{2}$

2) produit pur cristallisé de référence : d $\mathrm{CaCO}$, cristaux extraits du bois e et $\mathrm{f}$.

FiG. 14. - Poxitive prints of $X$-ray diffraction spectra

1) pure cristallized products : a $\mathrm{K}_{2} \mathrm{CO}_{2}$

b $\mathrm{MgCO}_{3}$

c $\mathrm{CaCO}_{3}$

2) pure cristallized product : d $\mathrm{CaCO}_{3}$ cristals extracted from wood of Poplar $\mathrm{e}$ and $\mathrm{f}$. 


\subsection{Microscope électronique à balayage.}

Nous avons utilisé un microscope électronique à balayage du type Stéréoscan au Laboratoire de M. le Professeur Baro à la Faculté des Sciences de Metz, avec l'aide de Mile Baltzinger et de M. Bhambri SushiL.

Cette technique, dérivée de la microscopie électronique (MenY et ChampignY, 1969 - Philibert, 1968), utilise comme faisceau incident des électrons primaires ayant une énergie maximale de $30 \mathrm{kV}$, et comme signal de détection, les électrons rétrodiffusés dans différentes directions, ainsi que les électrons secondaires de faible énergie émis par la surface de l'objet observé.

A cet effet, nous avons recouvert l'objet d'une très fine couche d'or-palladium par évaporation sous vide pour le rendre conducteur et permettre dans notre cas une meilleure observation de l'image.

Les signaux recueillis sont amplifiés et modulent la brillance du faisceau d'un tube cathodique d'observation. Ce faisceau balaye le tube en synchronisme avec le balayage de la sonde électronique sur l'échantillon.

L'image résultante a une apparence de relief à cause du contraste produit par la variation du nombre d'électrons recueillis liée à la microtopographie et aussi grâce à la très grande profondeur de champ du système. Elle apparaît sur l'écran cathodique et, avec un temps de pose de 40 à 100 secondes, elle peut être photographiée.

\section{Résultats}

Cette technique complémentaire permet de mieux visualiser le phénomène étudié, comme le montrent les figures 15 et 16, ainsi que les figures 8 et 9 insérées plus haut.

\section{3. - ANALYSE QUANTITATIVE}

\section{1. - Exposé des buts poursuivis}

L'analyse qualitative nous a permis d'identifier, de localiser et de dénombrer les cristaux présents, cerne par cerne. De nombreux auteurs, dont J. GAGNAIRE (1968) et RaLsTon et Prince (1963) ont déjà étudié globalement la distribution des ions minéraux dans les différentes parties des arbres feuillus ou résineux.

Nous avons cherché à aller plus loin en procédant à une analyse quantitative, cerne par cerne, de l'ion $\mathrm{Ca}^{+}$et des autres éléments majeurs susceptibles de l'accompagner : $\mathrm{K}^{+}, \mathrm{Mg}^{++}$, et de l'anion phosphore exprimé en $\mathrm{P}_{2} \mathrm{O}_{5}$.

La connaissance de la distribution des ions minéraux dans chaque accroissement permet en effet :

- de suivre le niveau atteint par chacun des ions en fonction de l'âge du bois et de mettre en évidence les relations existant entre eux,

- de considérer si, du point de vue de ces teneurs, le groupe des années constituant 


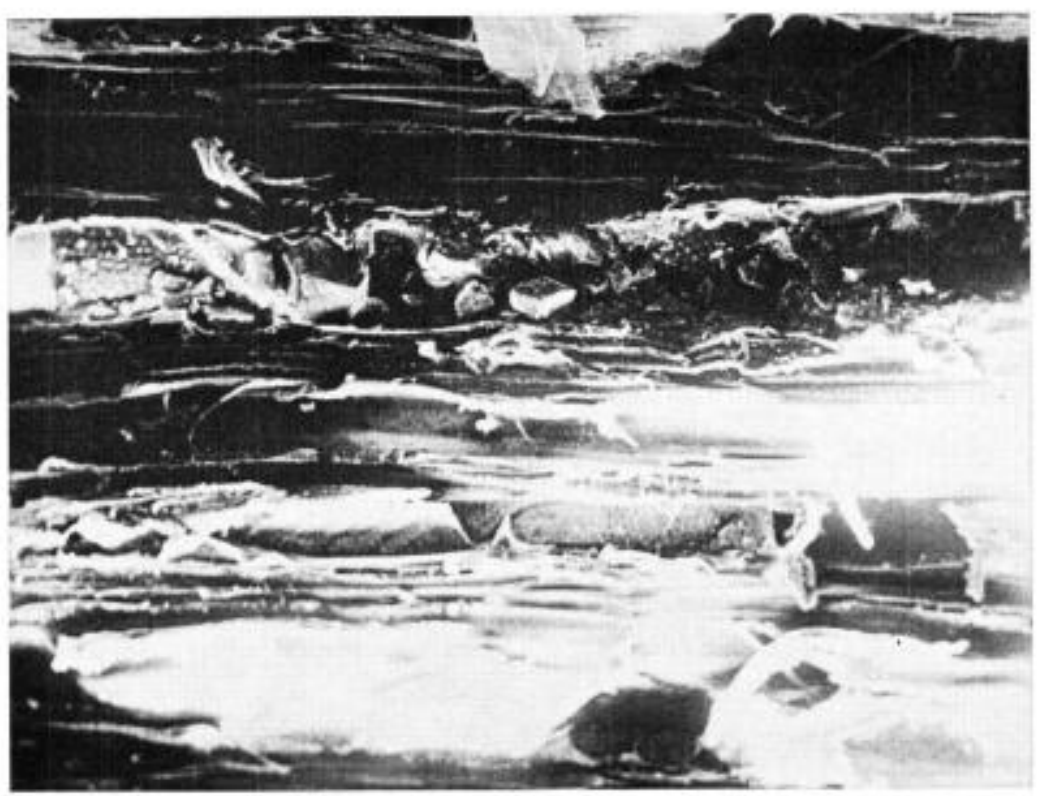

FIG. 15. - Deux lignes de vaisscaux voisines, Fune vide, Fautre remplie de $\mathrm{Ca} \mathrm{CO}_{2}$ parfaitement cristallisé, monocristal et translacide. $(X / 200)$.

Fic. 15. - Two adjacent lines of vessels, one empty, the other filled with $\mathrm{Ca} \mathrm{CO}_{3}$ perfectly crystallized, monocrystal and translacient. $(X / 200)$.

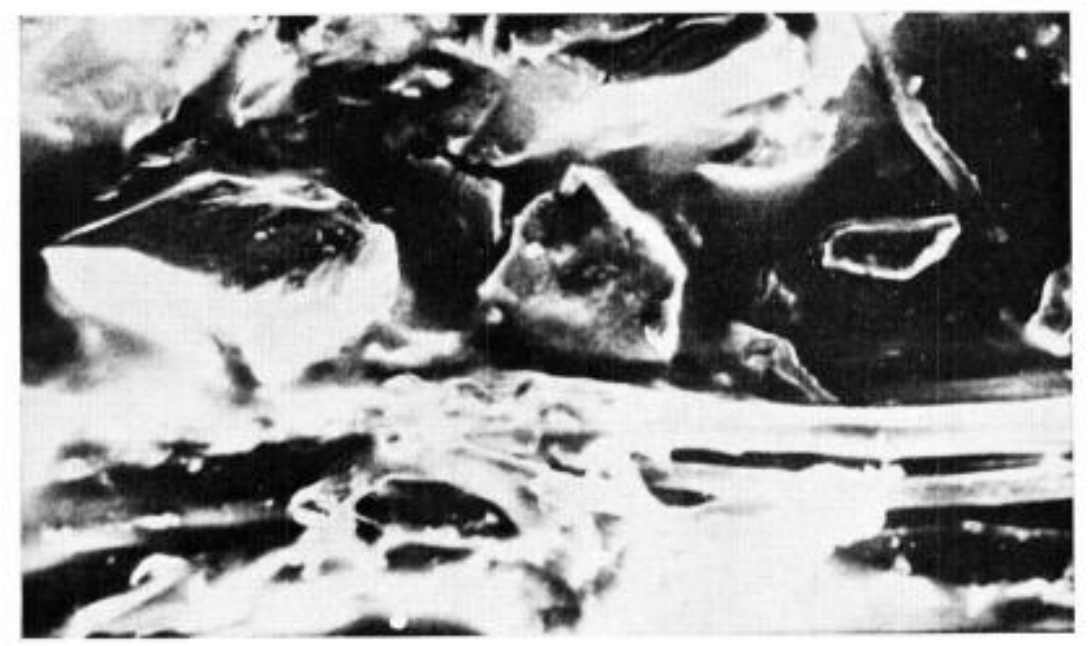

Fig. 16. - Fragments de cristaux brisés par le passage de la lame de microtome. Les cristaux ont des formes géométrigues très nettes. $(X 3000)$.

FiG. 16. - Crystals fragments broken by the microtome blade. They have very marked geometrical shapes. ( $x 3000)$. 
le bois d'aubier est fondamentalement différent du groupe des années formant le bois de cœur ou duramen coloré.

Il nous a êté possible, en effet, de constater sur des radiographies et à l'aide du comptage des vaisseaux obstrués des différences importantes entre ces deux groupes (voir figures 6 et 7).

\section{2. - Matériel et méthode}

\subsection{Matériel et origine du prélèvement.}

Le Populetum de Velaine-sous-Amance posséde un sol du type sol-brun lessivé à pseudo-gley. Le plancher de la nappe est généralement assez proche de la surface. La hauteur de celle-ci est variable et même peut submerger le sol. La roche-mère est constituée de limon sur marne. Le taux de saturation en surface atteint $50 \%$, en profondeur $100 \%$ au voisinage des marnes; en conséquence, le sol est bien pourvu en éléments $\mathrm{Ca}^{++}, \mathrm{Mg}^{++}, \mathrm{K}^{+}$; il $\mathrm{n}^{\prime} \mathrm{y}$ a jamais de calcaire,

Nos échantillons ont été prélevés sur un Populus trichocarpa de 15 ans, d'une part, 3 jeunes plants de la même espèce et 3 Populus robusta de 13 ans, d'autre part.

Les premiers ont étê utilisés comme matériel exploratoire, les autres pour l'analyse chimique quantitative proprement dite.

\subsection{Méthodes.}

\subsection{Echantillonnage exploratoire.}

Dans le bois de Populus trichocarpa " Fritzi Pauley " adulte, à l'aide de clichés radiographiques aux rayons $\mathrm{X}$, nous avons sélectionné des échantillons de bois d'aubier sans cristaux et de bois de coeur contenant des cristaux.

Ces échantillons débités dans les mêmes conditions pèsent approximativement 1 gramme, avec cette différence que nous avons circonscrit pour le bois de coxur le plus possible de zones contenant les inclusions minérales.

De ce fait, les teneurs ont pue être artificiellement augmentées, et cela dans le but de faire mieux ressortir les rapports existant entre les différents ions au cours d'analyses exploratoires.

\subsection{Analyse quantitative proprement dite.}

Les jeunes plants de Populus trichocarpa âgés, l'un de 3 ans et les deux autres de 2 ans, ont été étudiés à chaque entre-nœud sur des sections entières afin de confirmer l'augmentation des sels minéraux apportés par la présence de cristaux (voir figures 2 et 3).

Sur les Populus robusta (arbres I, II et III de notre étude), nous avons prélevé des sections entières de chaque arbre dans la bille de pied, que nous avons découpées selon chaque accroissement annuel. Les limites de cernes ont èté précisées à l'aide de clichés radiographiques aux rayons X, qui nous ont par ailleurs révélé la présence de vaisseaux obstrués sur chaque section.

Ces accroissements annuels ont été numérotés de 1 à 13 à partir du cœur vers l'écorce.

Les échantillons ont été minéralisés dans des capsules en silice au four ả moufle à $500^{\circ} \mathrm{C}$.

Les cendres ont été reprises à l'acide perchlorique dilué au demi. La solution de passage spectrophotométrique a èté amenée à la normalité I $\mathrm{N}$ en acide perchlorique : $\mathrm{HClO}_{*}$

Le calcium et le potassium ont été déterminès par spectrophotométrie d'émission, le magnésium par spectrophotométrie d'absorption atomique, le phosphore par colorimétrie au réactif vanadomolybdique.

\section{3. - Résultats}

\subsection{Analyse exploratoire.}

Les résultats sont donnés ci-dessous en $\%$ pour le $\mathrm{P}_{2} \mathrm{O}_{5}$ et en milliéquivalent $/ 100 \mathrm{~g}$ de bois sec pour les cations. 
TABLEAU 2

\begin{tabular}{|c|c|c|c|c|c|c|c|c|}
\hline \multirow[t]{2}{*}{$\mathrm{N}^{0}$} & \multicolumn{4}{|c|}{$\begin{array}{l}\text { Echantillons de bois d'aubier } \\
\text { sans cristaux }\end{array}$} & \multicolumn{4}{|c|}{$\begin{array}{l}\text { Échantillons de bois de cour } \\
\text { avec cristaux }\end{array}$} \\
\hline & $\mathrm{Ca}^{++}$ & $\mathrm{K}^{+}$ & $\mathrm{Mg}^{++}$ & $\mathrm{P}_{2} \mathrm{O}_{4}$ & $\mathrm{Ca}^{++}$ & $\mathrm{K}^{*}$ & $\mathrm{Mg}^{*+}$ & $\mathrm{P}_{2} \mathrm{O}_{2}$ \\
\hline 1 & 70 & 33 & 1,66 & 0.40 & 2000 & 120 & 16,2 & 0,43 \\
\hline 2 & 63.5 & 26 & 1,83 & 0,50 & 1865 & 83 & 19,1 & 0,56 \\
\hline 3 & 68 & 30 & 1,33 & 0.53 & 1920 & 113 & 20,4 & 0,45 \\
\hline 4 & 76,5 & 19 & 1.50 & 0.67 & 2015 & 123 & 17,5 & 0,44 \\
\hline 5 & 65 & 19 & 1,00 & 0,53 & 1865 & 93 & 16.6 & 0,44 \\
\hline 6 & 65 & 25 & 1,33 & 0,53 & 2310 & 126 & 15,4 & 0.40 \\
\hline Moyenne & 68 & 25 & 1,44 & 0,52 & 1995 & 109 & 17,5 & 0,45 \\
\hline
\end{tabular}

Echantillons de bois daubier sans cristaux - samples of sapwood without crystals

Echantillons de bois de cceur avec cristaux = samples of heartwood with crystals

Moyenne $=$ mean value

$\mathrm{Ca}^{++}, \mathrm{K}^{+}$et $\mathrm{Mg}^{++}$sont exprimés en meq $/ 100 \mathrm{~g}$ de bois $\mathrm{sec}=\mathrm{Ca}^{++}, \mathrm{K}^{+}$and $\mathrm{Mg}^{++}$are expressed in milliequivalent per hundred grams ovendry weight of wood.

$\mathrm{P}_{2} \mathrm{O}_{3}$ est exprimé en $\%$ de bois sec $=\mathrm{P}_{2} \mathrm{O}_{3}$ is expressed in $\%$ ovendry weight of wood.

Pour les ions $\mathrm{Ca}^{++}, \mathrm{Mg}^{++}, \mathrm{K}^{+}$, les différences sont évidentes et les augmentations sont liées à la présence des cristaux dans le bois de cœur. Pour le phosphore sous forme de $\mathrm{P}_{2} \mathrm{O}_{5}$, on constate une diminution significative lorsque I'on passe de l'aubier au bois de cour ; bien que celui-ci ait été sélectionné par la présence de cristaux, on peut déjà en déduire que le calcium n'est pas fixé sous forme de phosphate.

II est à noter que ce sont là des échantillons choisis après examen à l'aide de radiographies ; en l'absence de contrôle préliminaire, ils auraient pu constituer des prises d'essais pour la détermination des taux de cendres et des analyses minérales, mais les ordres de grandeur des résultats auraient alors été modifiés.

\subsection{2. Étude des jeunes plants.}

3.321. Tableau des résultats.

TABLEAU 3

\begin{tabular}{|c|c|c|c|}
\hline & $\begin{array}{l}\text { années } \\
1+2+3\end{array}$ & $\begin{array}{l}\text { années } \\
1+2\end{array}$ & anné \\
\hline $\begin{array}{l}\text { Plant de } 3 \text { ans }= \\
\qquad \begin{array}{l}\mathrm{Ca}^{++} \\
\mathrm{K}^{+} \\
\mathrm{P}_{2} \mathrm{O}_{3}\end{array}\end{array}$ & $\begin{array}{r}19,00 \\
7,60 \\
1,25\end{array}$ & $\begin{array}{l}7.20 \\
2.48 \\
0.87\end{array}$ & $\begin{array}{r}10,30 \\
5,30 \\
1,67\end{array}$ \\
\hline $\begin{array}{c}\text { Plant de } 2 \text { ans, } \mathrm{n}^{0} \mathrm{I}- \\
\mathrm{Ca}^{++} \\
\mathrm{K}_{\mathrm{P}_{2}} \mathrm{O}_{3}\end{array}$ & 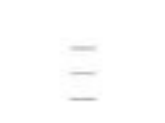 & $\begin{array}{l}8,64 \\
2,39 \\
1,89\end{array}$ & $\begin{array}{l}8,44 \\
3,57 \\
1,25\end{array}$ \\
\hline 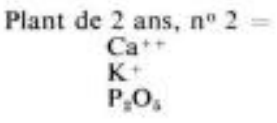 & E & $\begin{array}{l}8,40 \\
2,54 \\
1,68\end{array}$ & $\begin{array}{r}10,70 \\
3,80 \\
1,72\end{array}$ \\
\hline
\end{tabular}


Plant de 3 ans -3 year old plant

Plant de 2 ans $=2$ year old plant

annees $1+2+3-$ rings $1+2+3$

$\mathrm{Ca}^{++}$et $\mathrm{K}+$ sont exprimés en meq $/ 100 \mathrm{~g}$ de bois sec $-\mathrm{Ca}^{++}$and $\mathrm{K}+$ are expressed in milliequivalent per hundred grams ovendry weight of wood.

$\mathrm{P}_{2} \mathrm{O}_{5}$ est exprimé en $\%$ de bois sec $=\mathrm{P}_{2} \mathrm{O}_{5}$ is expressed in $\%$ ovendry weight of wood.

\subsection{Discussion.}

Sur des trẻs jeunes plants, nous constatons les mêmes phénomènes, à savoir :

- une augmentation simultanée des teneurs $\mathrm{Ca}^{++}$et $\mathrm{K}+$ comme le laissaient prévoir les accumulations de cristaux (figures $\mathrm{N}^{\circ s} 2$ et 3 du plant de 3 ans), étudié.

- une similitude des teneurs en phosphore sous forme $\mathrm{P}_{2} \mathrm{O}_{5}$ pour chaque entre-næud

En première et deuxième année, le $\mathrm{Ca}^{++}$est abondant et il n'y a pas de cristaux ; on en déduit que, dans cette zone périphérique où la circulation de sève est abondante, il se trouve sous forme, soit de sels minéraux solubles, soit de sels organiques solubles, soit encore engagé dans la matière organique.

En troisième année, une partie importante du calcium est en plus précipitće sous forme de carbonate de calcium.

\subsection{3. - Etude des sections entières de Populus robusta.}

L'interprétation des figures 17 à 23 permet de dégager trois phénomènes principaux :

- l'identité du comportement des 3 arbres,

- la répartition en trois ensembles distincts d'années recouvrant le voisinage du cambium, la partie de l'aubier et le bois de cour,

- I'existence d'un antagonisme entre le groupe des cations $\mathrm{Ca}^{++}, \mathrm{Mg}^{++}, \mathrm{K}+$ et le $\mathrm{P}_{2} \mathrm{O}_{5}$, et une opposition classique aubier-bois de ccur.

\subsection{Vue d'ensemble et comparaison graphique des trois arbres.}

Il est remarquable de constater (figures $17,18,19,20$ ) que la répartition des ions $\mathrm{Ca}^{++}$, $\mathrm{Mg}^{++}, \mathrm{K}^{+}$et du phosphore sous forme $\mathrm{P}_{2} \mathrm{O}_{5}$ est très semblable pour les trois arbres I, II et III sur l'ensemble des accroissements annucls étudiés (voir annexe 2).

Pour un élément donné et pour un cerne donné, les mesures faites sont toujours très voisines et forment un ensemble de courbes de variations très cohérent.

La représentation simultanée de toutes les courbes de variations pour le même arbre sur le même graphique et la faible dispersion des mesures permettent de repérer des zones de localisation des sels minéraux très marquées.

\subsection{Effet de la proximité du cambium.}

L'imprégnation par la sève de l'accroissement annuel le plus voisin du cambium provoque l'augmentation de la quantité des sels minéraux (figures $21,22,23$ ). On sait que la 


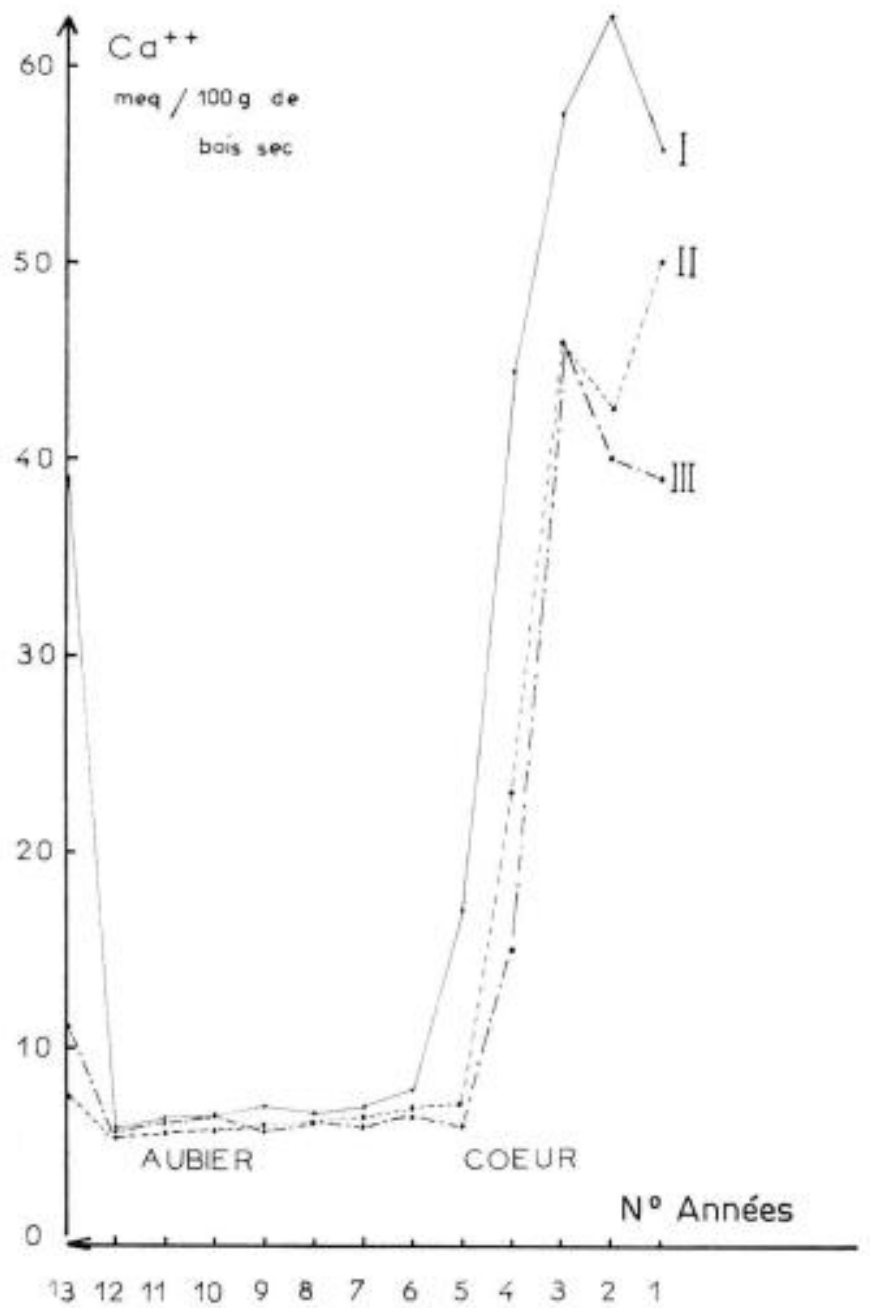

FIG. 17. - Courbes de variation année par année de la teneur en $\mathrm{Ca}^{++}$dans trois Populus robusta $1,11,111$. Distinction entre laubier of le carur.

Fig. 17. - Course, ring per ring, of the amount of $\mathrm{Ca}^{++}$in three Populus robusta I, II, III. Discrimination between sapwood and heartwowd.

sève a un $\mathrm{pH}$ inférieur à 7 ; donc ces sels se trouvent principalement sous des formes très solubles de sels-acides organiques et de phosphates acides substitués.

Nous donnons dans le tableau ci-dessous les valeurs correspondantes des concentrations en meq/100 g de bois sec dans le premier cerne sous écorce $\left(\mathrm{n}^{\circ} 13\right)$ et dans le cerne immédiatement voisin ( $\left.\mathrm{n}^{\circ} 12\right)$. C'est grâce aux analyses détaillées conduites cerne par cerne que nous avons pu mettre en évidence, pour la première fois semble-t-il, cette distinction. 
TABLEAU 4

\begin{tabular}{|c|c|c|c|}
\hline Ions & $\begin{array}{c}\text { 1ef cerne à partir } \\
\text { du cambium }\end{array}$ & $\begin{array}{l}2^{6} \text { cerne à partir } \\
\text { du cambium }\end{array}$ & $\Delta(C)$ \\
\hline $\begin{array}{c}\text { Arbre } \mathrm{I}: \\
\mathrm{Ca}^{++} \\
\mathrm{Mg}^{++} \\
\mathrm{K}^{+} \\
\mathrm{P}_{\mathrm{s}} \mathrm{O}_{\mathrm{s}}\end{array}$ & $\begin{array}{r}38,80 \\
4,51 \\
9,40 \\
2,16\end{array}$ & $\begin{array}{l}6,30 \\
1,74 \\
2,36 \\
0,92\end{array}$ & $\begin{array}{r}32,50 \\
+\quad 2,77 \\
+\quad 7,04 \\
+\quad 1,08\end{array}$ \\
\hline $\begin{array}{l}\text { Arbre II : } \\
\qquad \begin{array}{l}\mathrm{Ca}^{++} \\
\mathrm{Mg}^{++} \\
\mathrm{K}^{+} \\
\mathrm{P}_{2} \mathrm{O}_{3}\end{array}\end{array}$ & $\begin{array}{l}5,70 \\
1,22 \\
3,00 \\
0,93\end{array}$ & $\begin{array}{l}5,40 \\
1,44 \\
1,86 \\
0,97\end{array}$ & $\begin{array}{l}+\quad 0,30 \\
-\quad 0,22 \\
+\quad 1,14 \\
-\quad 0,04\end{array}$ \\
\hline 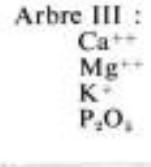 & $\begin{array}{r}11,50 \\
2,01 \\
4,81 \\
1,16\end{array}$ & $\begin{array}{l}5,90 \\
1,54 \\
2,08 \\
0.70\end{array}$ & $\begin{array}{r}+5,60 \\
+\quad 0,47 \\
+\quad 2,73 \\
+\quad 0,46\end{array}$ \\
\hline \multicolumn{4}{|l|}{$\begin{array}{c}2^{e} \text { cerne } \\
\mathrm{Ca}^{++}, \mathrm{M} \\
\text { liequivalent } \\
\mathrm{P}_{2} \mathrm{O}_{5} \text { est }\end{array}$} \\
\hline
\end{tabular}

Note, La différence constatée entre les deux premiers cernes montre que, dans le cas de prélèvement d'échantillons pour la détermination du taux de cendres, il convient de s'affranchir de la zone périphérique de la section retenue.

En effet, on atteint dès le deuxième accroissement annuel la valeur moyenne observée pour les cernes constituant la zone de l'aubier.

\subsection{Effet de Taubier.}

Le caractère essentiel du bois d'aubier est d'être homogène vis-à-vis des concentrations des constituants minéraux.

On n'enregistre que de faibles variations autour du niveau moyen de chaque palier de concentration pour chaque ion tout au long des cernes successifs composant le bois d'aubier, du $\mathrm{n}^{\circ} 12$ au $\mathrm{n}^{\circ} 6$.

Les ions $\mathrm{Ca}^{++}, \mathrm{Mg}^{+}, \mathrm{K}+$ sont sous forme soluble, amenés par la sève et aussi sous forme insoluble et cristallisée, essentiellement sous forme de $\mathrm{CaCO}_{3}$, comme on a pu les dénombrer au cours des comptages précédents (voir figures 6 et 7 et tableau 1).

Le phosphore est en majeure partie sous forme de phosphates acides substitués dans la sève brute qui imprègne l'aubier. Le phosphore sous forme $\mathrm{P}_{2} \mathrm{O}_{5}$ atteint $1 \%$ dans le bois d'aubier. 


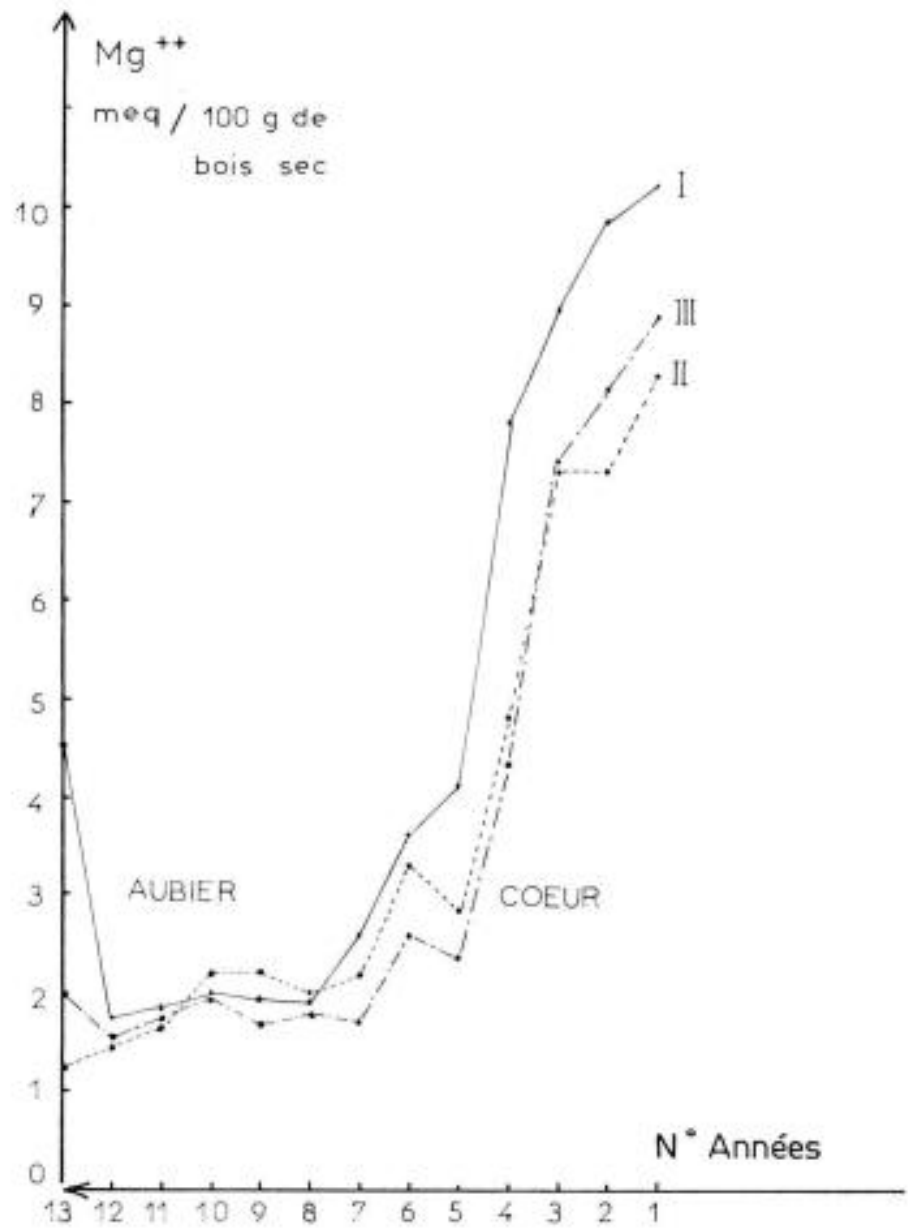

FiG. 18. - Courbes de variation année par année de la teneur en $M g^{+++}$dans trois Populus robusta $I, I I, I I I$. Distinction entre Paubier ef le caur.

Fig. 18. - Course, ring per ring, of the amount of $\mathrm{Mg}^{++}$, in three Populus robusta I, II, III. Discrimination between sapwood and heartwood.

3.334. Effet du bois de caur.

L'examen en séquence des accroissements annuels montre une augmentation brutale des concentrations en cations sur un espace minimum de deux années.

\section{- Cation $\mathrm{Ca}^{++}$:}

L'accumulation a son effet maximum pour le calcium : $\mathrm{Ca}^{++}$. La moyenne du palier commun aux trois arbres est de $6 \mathrm{meq}$ pour $100 \mathrm{~g}$ de bois sec pour l'aubier.

On observe sur deux ans une augmentation brutale de $\mathrm{Ca}^{++}$en meq égale au moins à cing fois la valeur de ce palier (voir tableau page 94). 


\begin{tabular}{c|c|c|c}
\hline No arbre & Cerne $\mathrm{n}^{\circ} 5$ & Cerne $\mathrm{n}^{\circ} 3$ & $\Delta\left(\mathrm{Ca}^{+*}\right)$ meq \\
\hline II & 16,8 & 57,7 & $+40,9$ \\
III & 7,2 & 35,6 & $+28,4$ \\
$+41,3$ \\
\hline \hline
\end{tabular}

Les autres années du bois de cour sont caractérisées par des concentrations très élevées en $\mathrm{Ca}^{++}$.

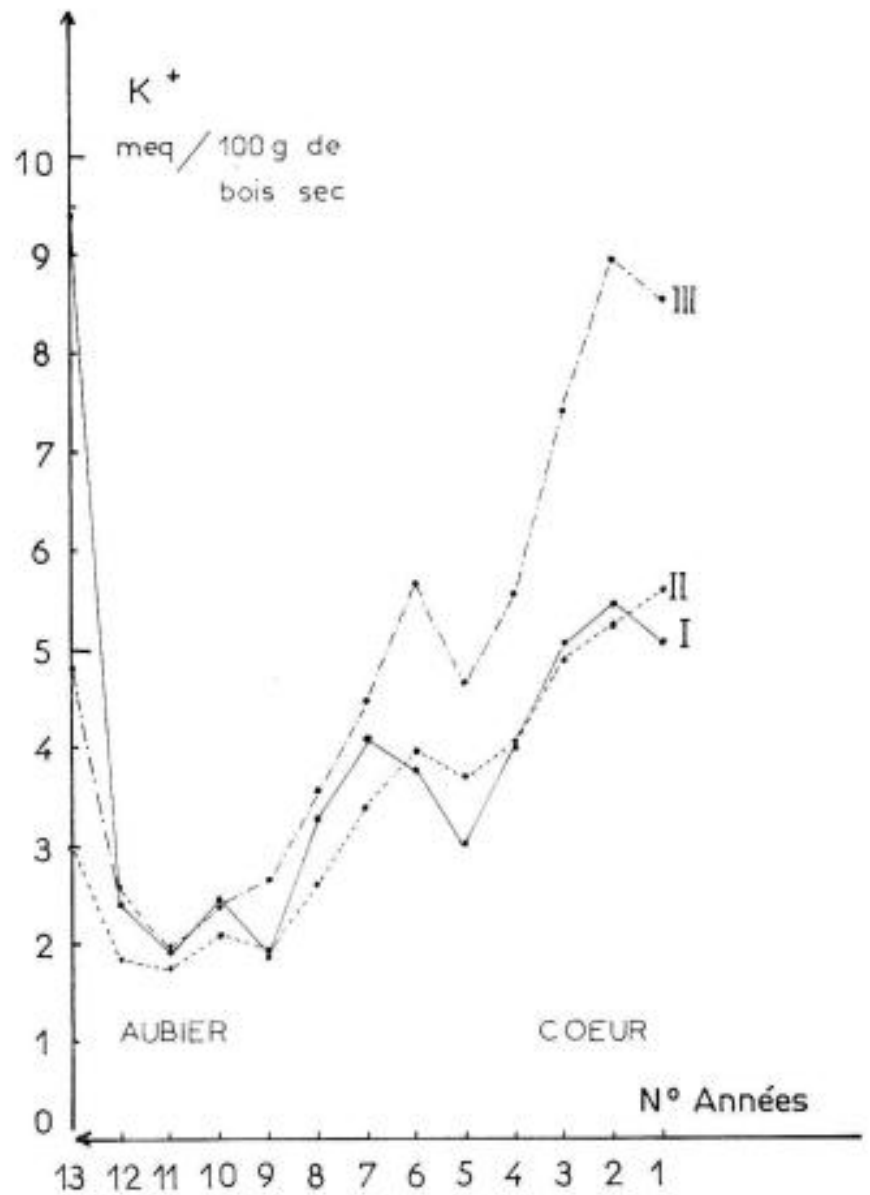

Fıo. 19. - Courbes de variation année par année de la teneur en $K^{*}$ dans trois Populus robusta I, II, III. Distinction entre Taubier et le carar.

F10. 19. - Course, ring per ring, of the amount of $\mathrm{K}^{+}$, in three Populus robusta ${ }^{2} I, I I, I I$. Discrimination between sapwood and hearfwood. 


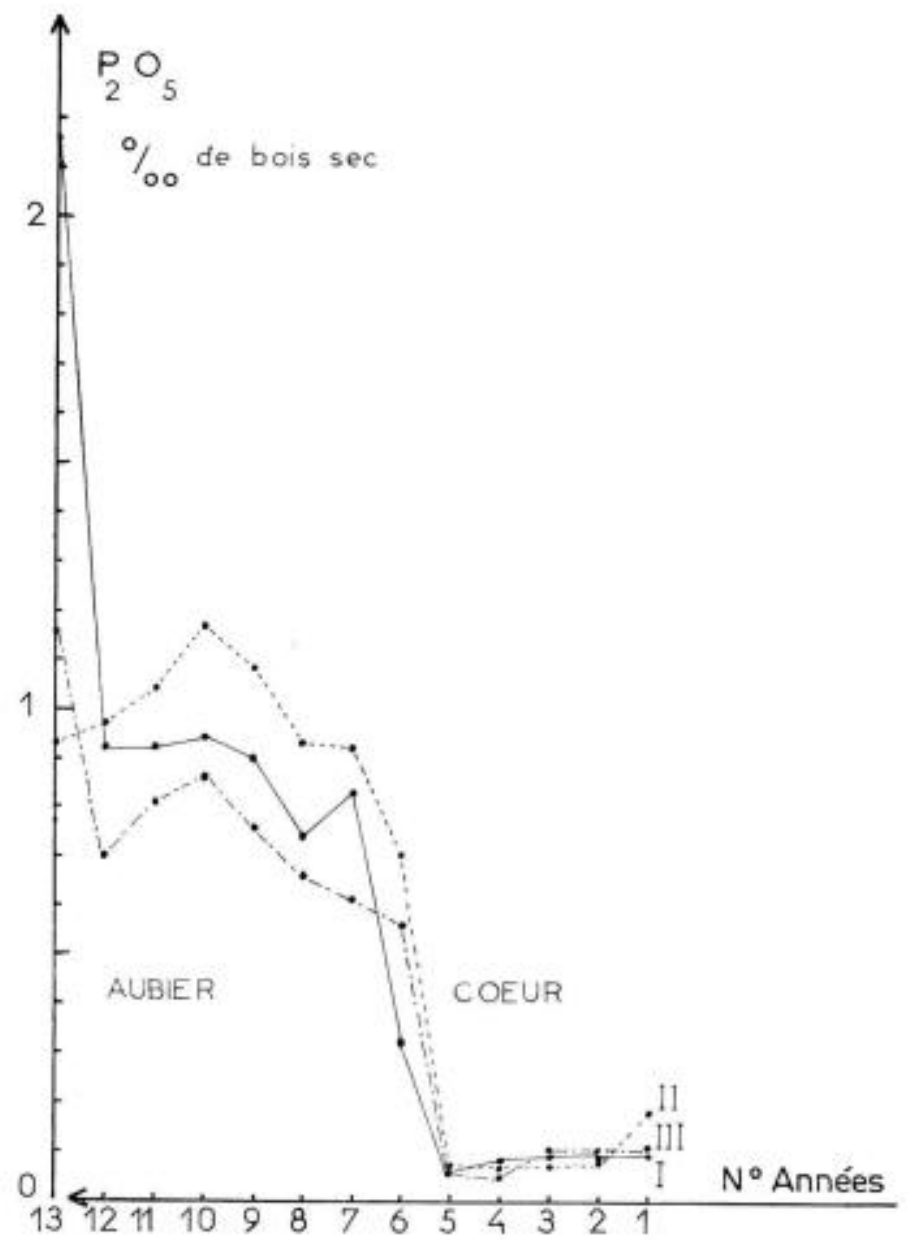

FIG. 20. - Courbes de variation année par année de la teneur en $P_{2} O_{3}$ dans trois Populus robusta $I, I I, I I I$. Distinction entre laubier et le caur.

FIG. 20. - Course, ring per ring, of the amount of $\mathrm{P}_{2} \mathrm{O}_{5}$ in three Populus robusta $I, I I, I I I$. Discrimination between sapwood and heartwood.

\section{- Cation $\mathrm{Mg}^{++}$:}

L'accumulation du $\mathrm{Mg}^{++}$est moins caractéristique, mais suit les mêmes lois que celle de $\mathrm{Ca}^{++}$.

Le palier « bois d'aubier w existe, mais il est ici moins constant. La concentration en $\mathrm{Mg}^{++}$augmente, en effet, d'une manière progressive lorsque l'on se rapproche du bois de cœur. Cependant, l'accumulation de $\mathrm{Mg}^{+*}$ entre les cernes $\mathrm{n}^{\circ} 5$ et $\mathrm{n}^{\circ} 3$ est très nette, comme pour le $\mathrm{Ca}^{++}$. 


\begin{tabular}{c|c|c|c}
\hline \hline No arbre & Cerne n'5 & Cerne $\mathrm{n}^{\circ} 3$ & $\Delta\left(\mathrm{Mg}^{++}\right)$ \\
& & & \\
\hline II & 4,10 & 8,93 & $+4,83$ \\
III & 2,81 & 7,30 & $+4,49$ \\
& 2,36 & 7,92 & $+5,56$ \\
\hline \hline
\end{tabular}

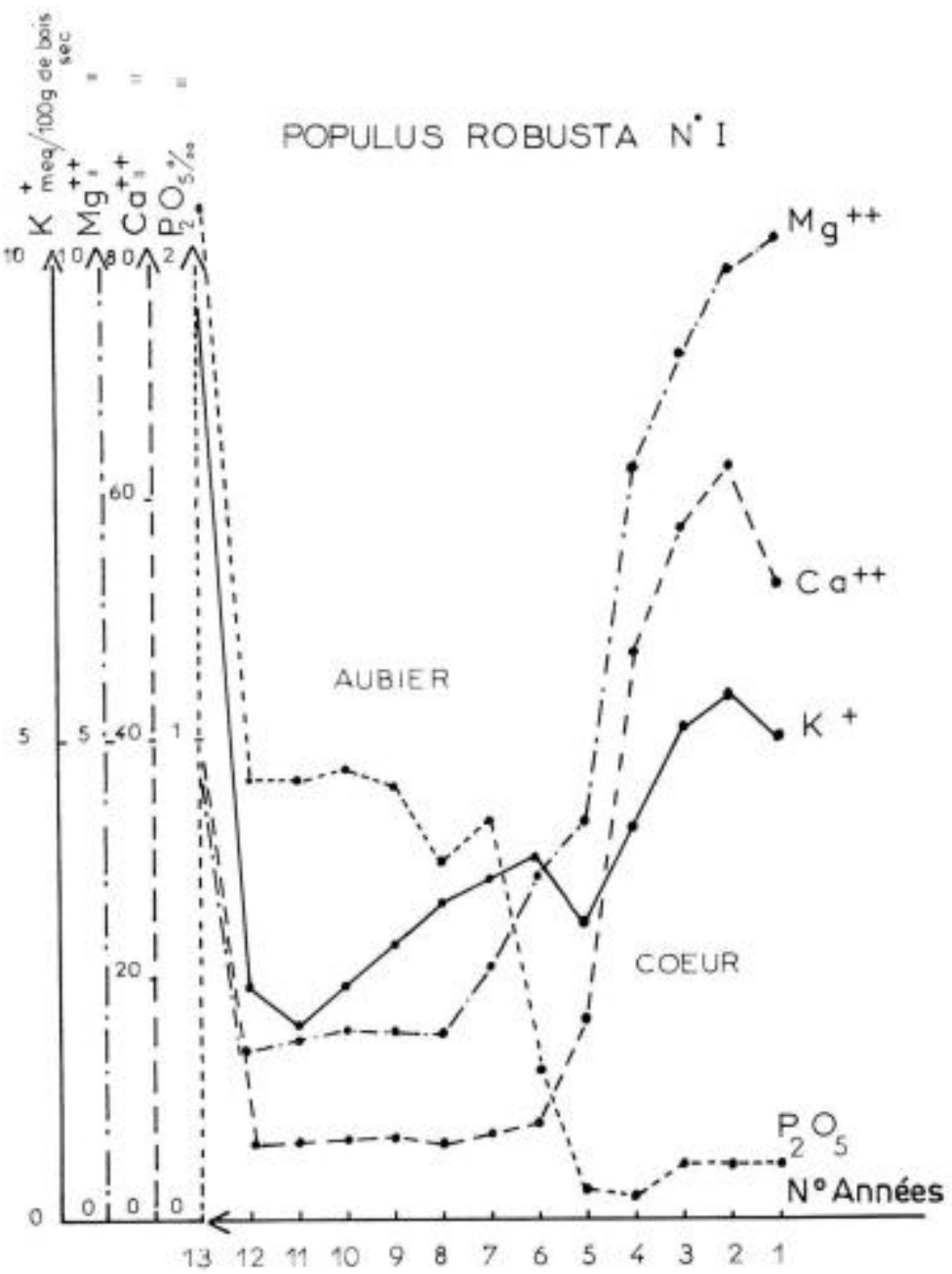

FIG. 21. - Courbes de variation comparées du groupe des cations $\mathrm{Ca}^{+}, \mathrm{Mg}^{++}, \mathrm{K}^{+}$et de lanion $\mathrm{P}_{2} \mathrm{O}_{5}$ dans l'aubier et le cour - Populus robusta $n^{0} I$.

FIG. 21. - Comparative nariations of group of cations $\mathrm{Ca}^{++}, \mathrm{Mg}^{++}, \mathrm{K}$ and of anion $\mathrm{P}_{2} \mathrm{O}_{3}$ observed in the sapwood and in the heartwood - Populus robusta $I$. 


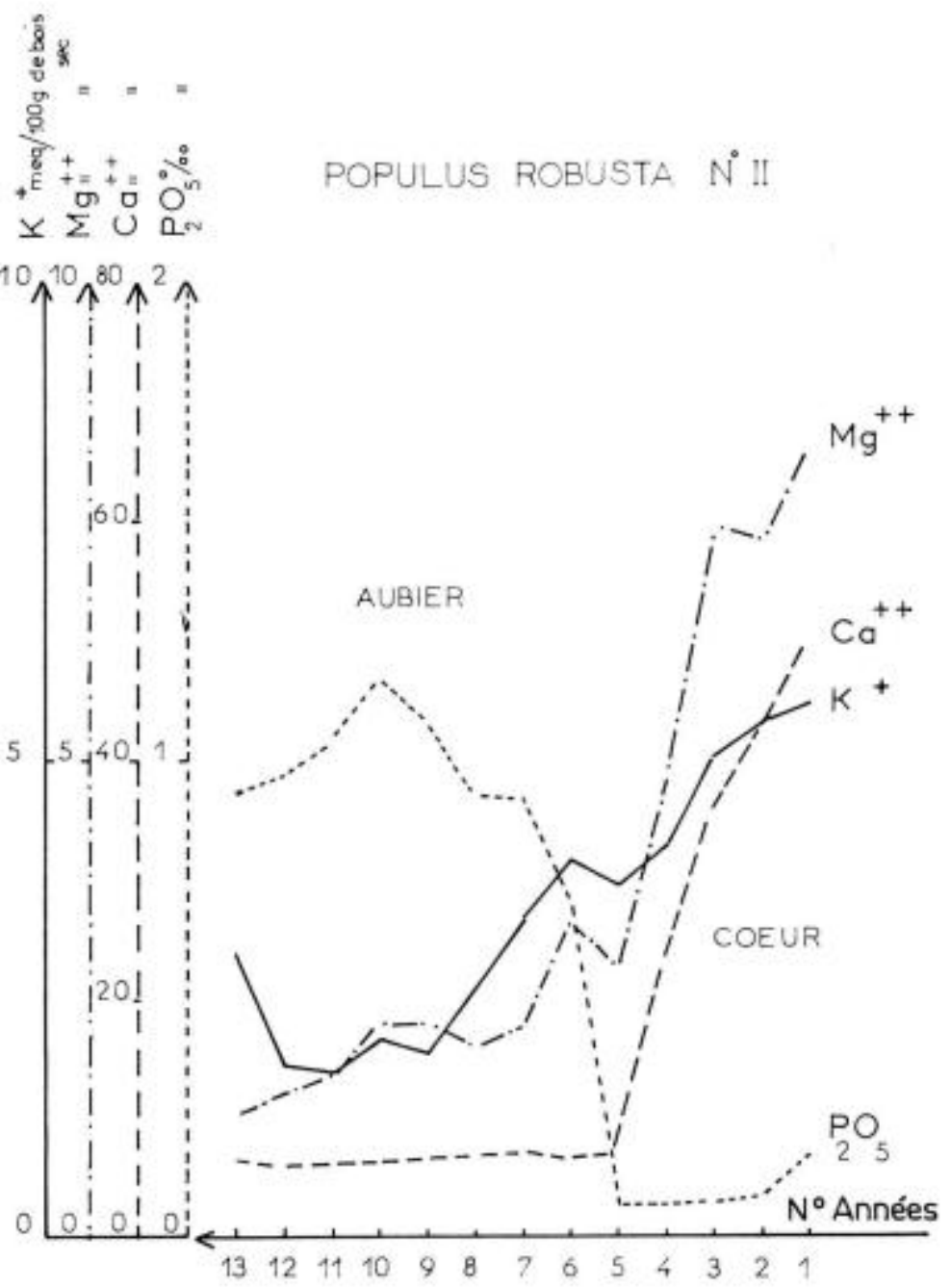

FiG. 22. - Courbes de variation comparées da groupe des cations $\mathrm{Ca}^{++}, \mathrm{Mg}^{++}, \mathrm{K}^{+}$et de l'anion $\mathrm{P}_{2} \mathrm{O}_{5}$ dans l'aubier er le caur - Populus robusta $n^{\circ} \mathrm{II}$.

Fic. 22. - Comparative variations of group of cations $\mathrm{Ca}^{++}, \mathrm{Mg}^{++}, \mathrm{K}^{+}$and of anion $\mathrm{P}_{2} \mathrm{O}_{3}$ observed in the sapwood and in the hearfwood - Populus robusta $I I$.

\section{- Cation $\mathrm{K}^{+}$;}

L'accumulation de $\mathrm{K}^{+}$est plus continue que celle du $\mathrm{Mg}^{++}$et du $\mathrm{Ca}^{++}$.

Dans le bois d'aubier, les concentrations sont constantes pour les cernes $n^{0 s} 12,11$, 10,9 ; elles augmentent très progressivement jusqu'au cerne $\mathrm{n}^{\circ} 5$. Du cerne $\mathrm{n}^{\circ} 5$ au cerne $\mathrm{n}^{\circ} 3$, comme pour les autres cations, l'augmentation devient très significative. 


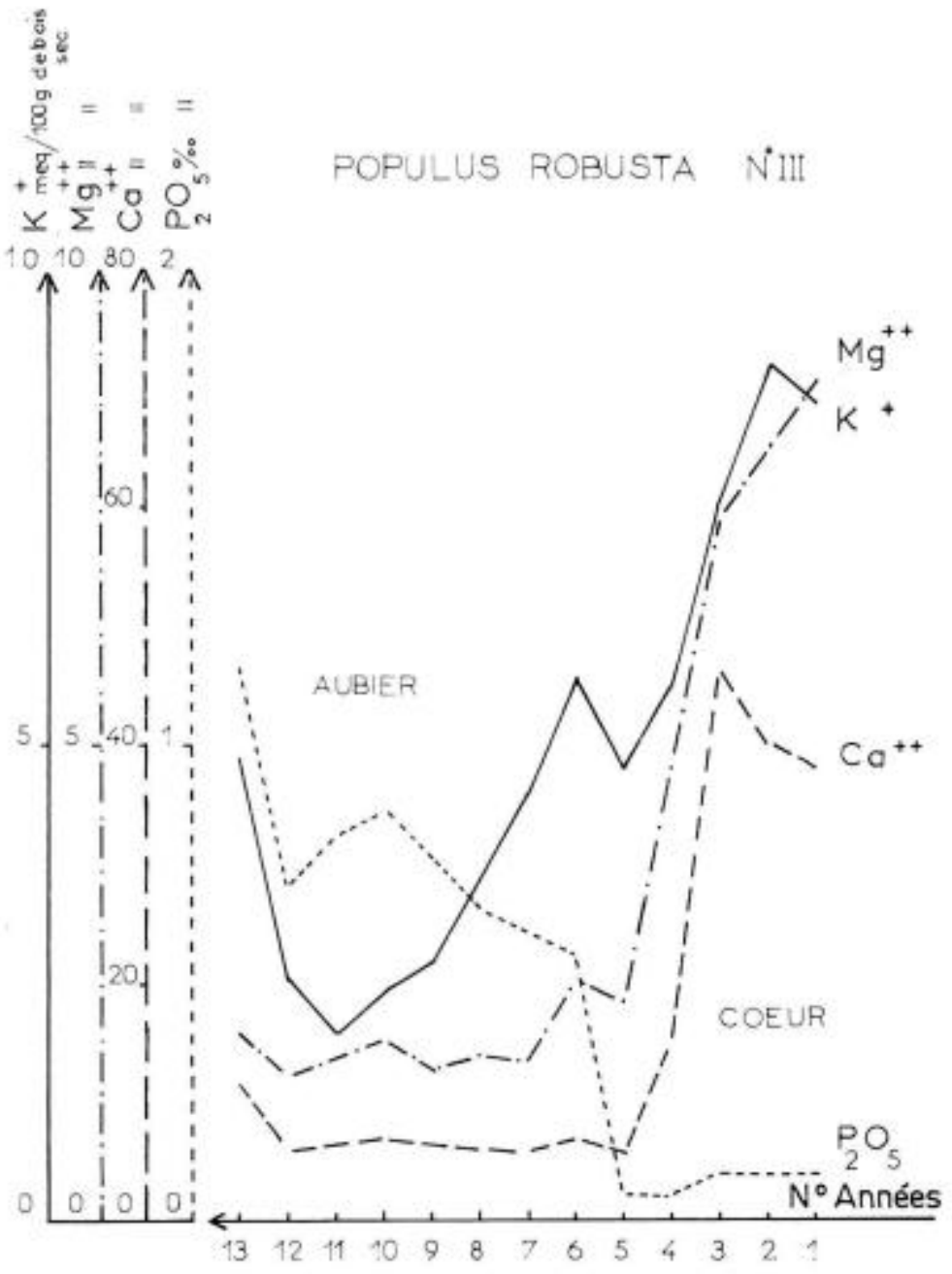

FiG. 23. - Courbes de variation comparées du groupe des cations $\mathrm{Ca}^{++}, \mathrm{Mg}^{++}, \mathrm{K}^{+}$et de lianion $\mathrm{P}_{2} \mathrm{O}_{3}$ dans l'aubier et le carur - Populus robusta $n^{\circ} \mathrm{III}$.

FiG. 23. - Comparative variations of group of cations $\mathrm{Ca}^{++}, \mathrm{Mg}^{+-}, \mathrm{K}^{+}$and of anion $\mathrm{P}_{3} \mathrm{O}_{3}$ observed in the sapwood and in the heartwood - Populus robusta III.

Pour les trois arbres, nous avons :

\begin{tabular}{|c|c|c|c|}
\hline $\mathrm{N}^{\circ}$ arbre & Cerne $n^{\circ} 5$ & Ceme no 3 & $\Delta\left(K^{+}\right)$ \\
\hline $\begin{array}{l}\text { I } \\
\text { III }\end{array}$ & $\begin{array}{l}2,98 \\
3,68 \\
4,67\end{array}$ & $\begin{array}{l}5,06 \\
4,89 \\
7,40\end{array}$ & 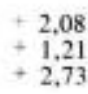 \\
\hline
\end{tabular}




\section{- Anion P}

Le phosphore a été le seul anion analysé choisi comme anion caractéristique participant à l'équilibre acido-basique du milieu.

Nous assistons à une diminution de concentration de l'élément lorsque nous passons de l'aubier au bois de cæur. Il existe, on l'a vu, un palier dans le bois d'aubier d'une moyenne de $1 \%$; dans le bois de cơur, oủ les apports de sève et les échanges ont cessé complètement, le palier n'est qu'à $0,1 \%$ on.

On peut penser que le phosphore, à ce niveau, compte tenu de sa faible concentration, se trouve uniquement engagé dans des complexes organiques.

Le point de rupture est encore le cerne $n^{\circ} 5$. Cette année peut servir de repère pour les phénomènes d'accumulations ou d'élutions minérales; nous lui avons donné le nom de cerne-charnière.

Notre discussion prendra donc appui sur le rôle charnière du cerne $\mathrm{n}^{\circ} 5$; c'est en effet à ce niveau que l'on passe de la zone du bois d'aubier au bois de caur, à la fois du point de vue de l'aspect visuel des colorations caractérisant les bois de ceur colorés et du point de vue du bilan minéral.

Pour l'anion $\mathrm{P}_{2} \mathrm{O}_{5}$, c'est le point d'arrivée aux concentrations constantes les plus basses, et pour les groupes des cations, le point de départ des accumulations importantes. Il existe une opposition entre $\mathrm{P}_{2} \mathrm{O}_{5}$ et le groupe de cations.

Cette opposition est plus nette si l'on exprime que le rapport cation sur anion est 60 fois plus fort dans le bois de cœur que dans le bois d'aubier.

Dans cette zone de passage, on assiste d'abord à une élution du phosphore contenu dans le bois d'aubier, à un départ du phosphore minéral au niveau des cernes $\mathrm{n}^{\circ \mathrm{s}} 7,6$ et 5 , puis à une accumulation rapide des cations au niveau des cernes $n^{05} 5,4$ et 3 .

En résumé, si l'on considẻre l'année de rang $n$ comptée à partir du cour comme année de transition entre l'aubier et le bois de cœur, les années $n+l$ et $n+2$ voient leur teneur en phosphore décroître jusqu'à l'année $n$, tout en conservant constantes leurs teneurs en cations, et les années $n-1, n-2$ voient leurs concentrations en cations augmenter rapidement tout en conservant constantes leurs teneurs en phosphore.

\section{4. - CONCLUSION}

Cette étude nous a paru nécessaire en raison de l'importance et du caractère général de l'existence de cristaux, constitués en majeure partie de carbonate de calcium, dans le bois des espèces du genre Populus.

L’ampleur du phénomène dépasse les simples remarques faites dans la littérature sur leur présence éventuelle dans les parties anormales du bois et indique, au contraire, que I'on a affaire à un processus physiologique normal des espèces considérées.

D'un point de vue qualitatif, à la suite des premières recherches, il se dégage les faits suivants :

— dans les trois sections étudiées à ce jour, seules les sections Aigeiros et Tacamahaca contiennent des cristaux ; la section Leuce en est dépourvue. Lorsqu 'une section contient des cristaux, toutes les espèces des groupes qui appartiennent à cette section en sont pour- 
vues. Nous pensons que ce caractère peut fournir un critère supplémentaire de détermination de la section à laquelle appartient un arbre considéré dans le genre Populus;

- lorsqu'un arbre contient des cristaux, on en trouve dans toutes les parties de l'arbre : branches, tiges, racines ; ils sont déposés dans les vaisseaux et les fibres du bois sain et normal. Ils sont essentiellement concentrés dans un cylindre central entourant la moelle où leur présence est constante et on les observe de façon aléatoire dans un cône excluant les cernes les plus extérieurs;

- ils peuvent atteindre des densités élevées et variables dans plus de la moitié du volume total de la tige.

La formation du bois de cœur et l'augmentation des teneurs en sels minéraux sont deux phénomènes très souvent rapprochés dans la littérature. Cependant, cette constatation a toujours été faite sur des arbres âgés, avec un bois de cœur constitué, sans tenir compte de l'aspect chronologique de l'apparition des dépòts cristallisés.

Or, nous avons montré sur de jeunes plants de Populus trichocarpa de trois ans, ainsi que sur Populus trichocarpa de quinze ans, la présence de cristaux abondants avec un duramen encore peu différencié.

Nous pouvons donc signaler, dans ce cas, un aspect nouveau concernant les relations existant entre la formation de bois de cour et l'augmentation de la teneur en sels minéraux, à savoir que l'accumulation des sels minéraux peut précéder dans le temps, et de beaucoup, la formation de celui-ci.

L'étude quantitative des cations $\mathrm{Ca}^{++}, \mathrm{Mg}^{++}, \mathrm{K}^{+}$et de l'anion phosphore sous forme $\mathrm{P}_{2} \mathrm{O}_{5}$, faite cerne par cerne, a permis de mettre en évidence sur Populus robusta les variations très fines des concentrations des divers ions et l'existence d'un cerne caractéristique de transition que nous avons appelé cerne-charnière entre le bois d'aubier et le bois de caur.

Les teneurs en phosphore, qui se situent sur un palier élevé dans les années du bois d'aubier, décroissent et se stabilisent au seuil le plus bas pour ce cerne-charnière ; ceci indique qu'il est épuisé en phosphore et qu'il commence à subir l'accumulation croissante des cations.

Il sépare deux bois aux teneurs minérales différentes et pourrait, de ce fait, apparaitre comme une limite ou un élément de différenciation analytique des zones aubier-bois de cœur.

Des études plus approfondies, actuellement en cours, portant sur un Populus trichocarpa analysé entre-nœud par entre-nœud et, pour chaque entre-nœud, cerne par cerne, nous permettront de confirmer ou d'infirmer l'existence de ce cerne limite tout au long de la tige.

Des échantillons plus nombreux provenant de Populetums différents français ou étrangers seront étudiés dans un travail ultérieur, afin de recouvrir les cinq sections du Genre avec un plus grand nombre d'espèces.

Enfin, pour tenter de mettre en évidence la généralité du phénomène, des études seront entreprises sur le bois d'aubier et le bois de caur d'arbres d'autres genres.

Si un cerne-charnière analytique apparaissait de façon constante, il pourrait servir de base à un procédé commode de délimitation de l'aubier dans les espèces à bois de cour non coloré. 


\section{REMERCIEMENTS}

Nous adressons nos sincères remerciements à :

M. LemorNe pour ses conseils et les échantillons collectés dans le Populetum de Velaine-sous-Amance dont il a la responsabilité,

M. Nahal, Professeur à la Faculté d’Agronomie, Université d'Alep-Syrie, et M. Rahme, Assistant. pour les échantillons fournis.

MM. Perriv et Thiriet pour la collecte, le débit et le façonnage des échantillons,

Mme Garros et M. Michel pour les travaux de coupes microscopiques de végétal et de radiographie, MM. Sutre et Michelot pour les analyses de chromatographie en phase gazeuse,

M. Gantots pour les travaux de microscopie électronique et aux rayons $\mathrm{X}$,

Mile BALtzinger et M. SushiL pour les observations au microscope électronique à balayage Stéréoscan,

\section{SUMMARY}

CRYSTALS OF CALCIUM CARBONATE IN THE WOOD OF THE GENUS POPULUS - CONSEQUENCES ON THE DISTRIBUTION OF MINERAL SUBSTANCES IN THE HEARTWOOD FORMATION.

The evidence of crystal deposit first arose from the visual examination and the densitometric recording of X-ray photographs of increment cores.

A survey concerning some species of 3 sections, report in table 1, shows that crystals are present in all the species of the section Aigeiros and Tacamahaca and absent in the species of the section Leuce.

From an investigation on young trees, it appears that the deposit of crystals begins as soon as the 3rd ring of the lst internode.

Crystals are present in the whole tree (fig. 4 and 5) excepted the first rings near the bark where the sap flow is abundant. The appendix 1 summarizes the number and the density per ring of the occluded vessels at 3 different levels.

The occlusion of vessels is sometimes partial, more often total $;$ in this case the crystals give a moulding of the vessel (see electromicroscope figures 8 and 9 where the print of 2 pits is apparent). Crystallisation occurs also within fibers (fig. 12) surrounding the filled vessels.

Several physical and physico-chemical methods have been used for the determination of crystals.

For ga chromatography several hundreds of thin sections were soaked in hydrochloric acid and the ga obtained were identified as carbon dioxyd (fig. 13).

From X-ray diffraction spectrum it appears (fig. 14) that crystals specially consist of calcium carbonate in association with minute quantities of other minerals.

Quantitative chemical analysis have been done for several cations : $\mathrm{Ca}^{++}, \mathrm{Mg}^{++}$and $\mathrm{K}^{+}$and for the Phosphorus measured in terms of $\mathrm{P}_{2} \mathrm{O}_{3}$.

For young trees table 3 shows that the mineral salts are only present in the 3 rd ring that is the ring where crystals were observed.

Three older trees were also investigated. It appears from fig. 17 to 20 that the variations for these trees were quite similar : the phosphorus is more abundant within the sapwood ; it decreases near the 5th ring and its dose becomes 10 times lower in the heartwood.

The results for the cations are quite opposite : high level in the heartwood, and low level is the sapwood (table 2, appendix 2 , and tables included in the text section 3334).

A particular attention is paid to this 5th ring which may be called hinge-ring from the point of view of the analytic separation of mineral substances.

Elsewhere the table 4 shows a sharp diminution of the cations from the lst to the 2 nd ring from the cambium, and a more questionable variation for the phosphorus.

Thus modifications of the ionic concentration if corroborated by further investigations might serve to distinguish sections of the genus Populus and to delimitate sapwood in species with non colored heartwood.

Elsewhere this study has shown that à conserver to litterature crystals in the genus Populus arise from a normal physiological process and not at all from defects or other abnormal phenomena. 


\title{
ZUSAMMENFASSUNG
}

\author{
DIE FESTSTELLUNG VON KAL.ZIUMKARBONATKRISTALLEN IM HOLZ DER GATTUNG POPULUS \\ - EINFLUSS AUF DIE MINERALIONENVERTEILUNG IM KERNHOLZ.
}

Es wurde eine systematische Untersuchung ūber das Auftreten von Kristallen im Holz von Jungpflanzen und Bäumen der Gattung Populus durchgeführt, nachdem an Röntgenaufnahmen von zahlreichen Bohrspanproben das Vorhandensein von Kristallen festgestellt worden war. Es wurde eine qualitative und quantitative Untersuchung der kristallinen Ablagerungen durchgeführt.

Die qualitative Analyse zeigt, dass in den Sektionen Aigeiros und Tacamahaca ständig Kristalle vorhanden sind, während diese in der Sektion Leuce vollständig fehlen. Die Anzahl der verstopften Gefässe wurde jahrringweise an vollständigen mikroskopischen Stammschnitten von Populus trichocarpa ausgezảhlt. Die Verteilungsmodalitäten der Ablagerungen in den Gefässen und Fasern des Stammes, der Zweige und der Wurzeln werden dargestellt. Die verschiedenen Analysenmethoden (Gas-Chromatographie, Röntgenspektrographie, klassische Elektronenmikroskopie und Stereoscan - Elektronenmikroskopie) haben ergeben, dass die Kristalle nahezu ausschliesslich aus Kalziumkarbonat bestehen.

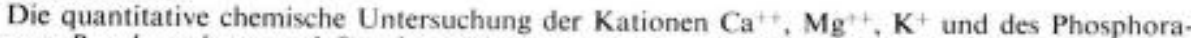
nions an Populus robusta und Populus trichocarpa erlaubte jahrringweise die erreichten Konzentrationen zu berechnen und die durch das Auftreten von Kristallen festgestellten Variationen zahlenmàssig zu belegen.

Desweiteren wurden an jedem Querschnitt analytisch drei Jahrringgruppen unterschieden :

- der erste Jahrring nach dem Kambium.

- das ubrige Spintholz,

- das Kernholz.

wobei ein Grenzjahrring festgestellt werden konnte, welcher bezüglich der lonenkonzentration einen Ubergang vom Splint zum Kernholz darstellt.

\section{RIASSUNTO}

MESSA IN EVIDENZA DI CRISTALLI DI CARBONATO DI CALCIO NEL LEGNO DEI PIOPPI - CONSEGUENZE SULLA RIPARTIZIONE DEI IONI MINERALI LEGATA ALLA FORMAZIONE DEL DURAME.

Una ricerca sistemàtica della presenza di cristalli nel legno del genere Populus su giovani piantine ed su alberi adulti fu intrapresa dopo la loro scoperta sui film esposti ai raggi $X$ durante le analisi di numerosi campioni ottenuti sotto la forma di carotine di sondaggio, colla trivella di Pressler.

I depositi cristallizzati furono analizzati qualitativamente e quantitativamente.

Lo studio qualitativo ha mostrato una presenza costante dei cristalli nelle sezioni Ageiros e Tacamahaca e una mancanza completa nella sezione Leuce. II numero dei vasi ostruiti fu determinato, anello a anello, usando sezioni radiali microscopiche, pezzi a pezzi, della superficia intera del fusto di Populus trichocarpa.

Le modalità della ripartizione dei depositi nei vasi e nelle fibre del tronco, dei rami e delle radici furono descritti.

Le analisi fatte mediante la chromatografia in fase gassosa, la spectroscopia ai raggi $\mathrm{X}$ e la microscopia elettronica classica e a analisi del tipo STEREOSCAN hanno dimostrate che $i$ cristalli erano per la maggiore parte formati dal carbonato di calcio.

Lo studio quantitativo chimico su Populus robusta e su Populus trichocarpa dei cationi Ca't. $\mathrm{Mg}^{++}, \mathrm{K}^{+}$e dell' anione fosforo a permesso di ciffrare anello a anello le concentrazioni raggiunte e le variazioni osservate colla presenza dei cristalli.

Inoltre, abbiamo separati analiticamente tre gruppi di anni su ogni sezione :

- Tanello vicino della zona cambiaria.

- la parte dell alburno.

- il durame,

ed abbiamo messi in evidenza un anello-cerniera che serve di transizione dal punto di vista delle concentrazioni in ioni tra Talburno e il durame. 


\section{RÉFÉRENCES BIBLIOGRAPHIQUES}

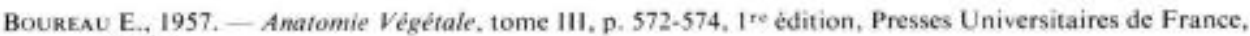
Paris.

EL.LIs E. L., 1967, - Minerals, crystals, and heartwood. XIVe Congrès IUFRO, Section 4I, Munich.

GaGNaIRE J., 1967. - Recherches sur la détection des pollutions radioactives par les végétaux. Thèse Docteur ès Sciences. Facultế des Sciences de l'Université de Grenoble.

KenNedY R. W., SAstry C. B. R., BArton G. M. et Elus E. L., 1968. - Crystals in the wood of the genus Abies indigenous to Canada and the United States. Canadian Journal of Botany, vol, $46, \mathrm{n}^{\circ} 10$. $1221-1228$

Meny L. et Champrony M., 1969, - Quelques applications métallurgiques du microscope èlectronique à balayage, J. Microscopic, 8, 663-676.

METCALFE C. R. et CHALK L., 1950, - Anatomy of Dicotyledons : leaves sten and wood in relation to taxonomy, with notes on economic use. Oxford, Clarendon Press, 2 vol.

MıA A. J., 1969, - Light and electron microscope studies of crystalline substances in Acer Saccharum mineral stain. Wood Science, vol, 2, n० 2, 120-124.

PANshin A. J. et de ZeEuw C., 1964. - Textbook of wood technology, vol, 1, p. 154, 155, 159, seconde édition, McGraw-Hill Book Company, New York.

Phil.anert J., 1968, - La microscopie électronique à balayage et ses applications métallurgiques. Revue de Physique Appliquée, tome III, décembre, p. 325-330.

POLGE H., 1966. - Erablissenent des courbes de variarion de la densité du bois par exploration denxitométrique de radiographies d'échantillons prélevés à la tariere sur des arbres vivants. Applications dans les domaines technologique et physiologique. Thèse Docteur ès Sciences Appliquées, Faculté des Sciences de l'Université de Nancy.

Polge H., 1967, - Première appréciation de la qualité du bois de Populus trichocarpa cultivé dans le Populetum de Velaine. Document à usage interne, Station de Recherches sur la Qualité des Bois du C.N.R.F.

Raiston C. W. et Privce. A. B., 1963. - Accumulation of dry matter and nutrients by Pine and Hardwood forests in the Lower Pledmont of North Carolina. Forest-Soil relationships in North America, p. 76-94, Youngberg, Corvallis.

Record S. J., 1927. - Occurrence of calcium carbonate deposits in woods. Tropical woods, n* 12, p, 22-26. 
ANNEXE ।

Niveau 3 mètres :

\begin{tabular}{|c|c|c|c|c|c|c|c|c|c|c|c|c|c|c|c|}
\hline $\begin{array}{l}N^{* 0} \text { des cernes de l'in- } \\
\text { tèrieur vers l'extérieur }\end{array}$ & 1 & 2 & 3 & 4 & 5 & 6 & 7 & 8 & 9 & 10 & 11 & 12 & 13 & 14 & 15 \\
\hline $\begin{array}{l}\text { Nombre de vaisseaux } \\
\text { obstrués ............ }\end{array}$ & 234 & 256 & 229 & 442 & 362 & 214 & 423 & 682 & 265 & 6 & 0 & 33 & 12 & 0 & 0 \\
\hline $\begin{array}{l}\text { Surface des cernes (en } \\
\left.\mathrm{cm}^{2}\right) \ldots \ldots \ldots \ldots \ldots \ldots\end{array}$ & 0,37 & 0,78 & 3,68 & 6.20 & 12,4 & 21,2 & 31,0 & 49,5 & 39,6 & 76,7 & 72,9 & 82,7 & 73,2 & 79,5 & 87,5 \\
\hline Densité moyenne ... & 632 & 328 & 62,2 & 71,2 & 29,2 & 10 & 13,6 & 13,7 & 6,7 & 0,07 & 0 & 0,40 & 0,16 & 0 & 0 \\
\hline
\end{tabular}

Niveau 9 mètres:

\begin{tabular}{l|c|c|c|c|c|c|c|c|c|c}
\hline $\begin{array}{l}\text { Numéros des cernes de l'in- } \\
\text { térieur vers l'extérieur ...... }\end{array}$ & 1 & 2 & 3 & 4 & 5 & 6 & 7 & 8 & 9 & 10 \\
Nbre de vaisseaux obstrués. & 357 & 474 & 430 & 36 & 50 & 4 & 0 & 0 & 0 & 0 \\
Surface des cernes (en $\mathrm{cm}^{2}$ ). & 2,15 & 5,28 & 20,7 & 33,9 & 25,9 & 34,2 & 36 & 37 & 74 & 43 \\
Densité moyenne ........... & 166 & 89,7 & 2,07 & 1,06 & 2 & 0,11 & 0 & 0 & 0 & 0 \\
\hline
\end{tabular}

Niveau 18 mètres: $n \quad \because *$

\begin{tabular}{l|c|c|c|c|c|c}
\hline $\begin{array}{l}\text { Numéros des cernes de l'inté- } \\
\text { rieur vers I'extérieur .......... }\end{array}$ & 1 & 2 & 3 & 4 & 5 & 6 \\
Nombre de vaisseaux obstrués. & 10 & 468 & 3 & 0 & 0 & 0 \\
Surface des cernes $\left(\mathrm{en} \mathrm{cm}^{3}\right.$ ) ... & 0,50 & 3,3 & 11,4 & 19 & 23,8 & 31,5 \\
Densité moyenne ............... & 20 & 142 & 0,26 & 0 & 0 & 0
\end{tabular}

Niveau $3 \mathrm{~m}=$ at level 3 meters. Niveau $9 \mathrm{~m}$ - at level 9 meters Niveau $18 \mathrm{~m}=$ at level 18 meters Numéros des cernes de l'intérieur vers l'extérieur - Number of the rings from the inner to the outer Nombre de vaisseaux obstrués $=$ Number of filled vessels

Surface des cernes $\left(\mathrm{cm}^{2}\right)=$ area of annual rings $\left(\mathrm{cm}^{2}\right)$

Densité moyenne $=$ average density 
ANNEXE 2

\begin{tabular}{|c|c|c|c|c|c|}
\hline $\mathrm{N}^{0}$ arbre & $\begin{array}{l}\text { Noa des cernes } \\
\text { de l'intérieur } \\
\text { vers l'extérieur }\end{array}$ & $\mathrm{P}_{2} \mathrm{O}_{s}$ & Calcium & Magnésium & Potassium \\
\hline 1 & $\begin{array}{r}1 \\
2 \\
3 \\
4 \\
5 \\
6 \\
7 \\
8 \\
9 \\
10 \\
11 \\
12 \\
13\end{array}$ & $\begin{array}{l}0,09 \\
0,09 \\
0,09 \\
0,08 \\
0,06 \\
0,32 \\
0,53 \\
0,14 \\
0,90 \\
0,94 \\
0,92 \\
0,92 \\
2,16\end{array}$ & $\begin{array}{r}55,6 \\
62,7 \\
57,7 \\
47,1 \\
16,8 \\
7,7 \\
6,9 \\
6,4 \\
6,8 \\
6,7 \\
6,4 \\
6,3 \\
38,8\end{array}$ & $\begin{array}{r}10,20 \\
9,85 \\
8,93 \\
7,80 \\
4,10 \\
3,61 \\
2,59 \\
1,91 \\
1,93 \\
2,01 \\
1,85 \\
1,74 \\
4,51\end{array}$ & $\begin{array}{l}5,04 \\
5,45 \\
5,06 \\
4,15 \\
2,98 \\
3,77 \\
4,16 \\
3,25 \\
1,96 \\
2,45 \\
1,87 \\
2,36 \\
9,40\end{array}$ \\
\hline II & $\begin{array}{r}1 \\
2 \\
3 \\
4 \\
5 \\
6 \\
7 \\
8 \\
9 \\
10 \\
11 \\
12 \\
13\end{array}$ & $\begin{array}{l}0,08 \\
0,09 \\
0,07 \\
0,07 \\
0,07 \\
0,70 \\
0,92 \\
0,93 \\
1,08 \\
1,17 \\
1,04 \\
0,97 \\
0,93\end{array}$ & $\begin{array}{r}49,8 \\
42,7 \\
35,6 \\
22,8 \\
7,2 \\
6,7 \\
6,9 \\
6,7 \\
6,5 \\
6,2 \\
5,9 \\
5,4 \\
5,7\end{array}$ & $\begin{array}{l}8,28 \\
7,30 \\
7,30 \\
4,79 \\
2,81 \\
3,30 \\
2,18 \\
2,01 \\
2,21 \\
2,22 \\
1,64 \\
1,44 \\
1,22\end{array}$ & $\begin{array}{l}5,60 \\
5,46 \\
4,89 \\
4,12 \\
3,68 \\
3,98 \\
3,40 \\
2,62 \\
1,93 \\
2,12 \\
1,76 \\
1,86 \\
3,00\end{array}$ \\
\hline III & $\begin{array}{r}1 \\
2 \\
3 \\
4 \\
5 \\
6 \\
7 \\
8 \\
9 \\
10 \\
11 \\
12 \\
13\end{array}$ & $\begin{array}{l}0,10 \\
0,10 \\
0,10 \\
0,05 \\
0,06 \\
0,56 \\
0,61 \\
0,66 \\
0,76 \\
0,86 \\
0,81 \\
0,70 \\
1,16\end{array}$ & $\begin{array}{r}39,1 \\
40,9 \\
47,2 \\
15,0 \\
5,9 \\
6,6 \\
6,0 \\
6,2 \\
5,8 \\
6,7 \\
6,3 \\
5,9 \\
11,5\end{array}$ & $\begin{array}{l}8,87 \\
8,14 \\
7,92 \\
4,32 \\
2,36 \\
2,59 \\
1,70 \\
1,72 \\
1,67 \\
1,94 \\
1,73 \\
1,54 \\
2,01\end{array}$ & $\begin{array}{l}8,53 \\
8,94 \\
7,40 \\
5,54 \\
4,67 \\
5,65 \\
4,47 \\
3,54 \\
2,65 \\
2,40 \\
1,96 \\
2,08 \\
4,81\end{array}$ \\
\hline
\end{tabular}

No arbre $=$ Number of the trees.

Nos des cernes de l'intérieur vers l'extérieur = Number of the rings from the inner to the outer.

$\mathrm{P}_{2} \mathrm{O}_{5}$ est exprimé en $\%$ de bois sec. $-\mathrm{P}_{2} \mathrm{O}_{\text {}}$ is expressed in $\%$ ovendry weight of wood.

$\mathrm{Ca}^{+}, \mathrm{Mg}^{+}$et $\mathrm{K}^{+}$sont exprimés en meq/100 de bois $\sec =\mathrm{Ca}^{+}, \mathrm{Mg}^{+}+$et $\mathrm{K}^{+}$are expressed in milliequivalent per hundred grams ovendry weight of wood. 